\title{
The Morphologies and Kinematics of Supernova Remnants
}

\author{
Laura A. Lopez • Robert A. Fesen
}

Received: 15 January 2018 / Accepted: 26 January 2018

\begin{abstract}
We review the major advances in understanding the morphologies and kinematics of supernova remnants (SNRs). Simulations of SN explosions have improved dramatically over the last few years, and SNRs can be used to test models through comparison of predictions with SNRs' observed large-scale compositional and morphological properties as well as the three-dimensional kinematics of ejecta material. In particular, Cassiopeia A - the youngest known core-collapse SNR in the Milky Way - offers an up-close view of the complexity of these explosive events that cannot be resolved in distant, extragalactic sources. We summarize the progress in tying SNRs to their progenitors' explosions through imaging and spectroscopic observations, and we discuss exciting future prospects for SNR studies, such as X-ray microcalorimeters.
\end{abstract}

\section{Introduction}

The predictive power of supernova explosion (SN) simulations has improved dramatically in the last few years. Three-dimensional simulations that properly handle energy-dependent neutrino transport obtain successful explosions via neutrino heating (Lentz et al 2015, Janka et al 2016, Roberts et al 2016, Müller et al 2017). These models are beginning to make testable predictions, such as the expected light curves for different mass progenitors, neutron star (NS) kick velocities, and large-scale compositional asymmetries (e.g., Wongwathanarat et al 2013a, Utrobin et al 2017, Wongwathanarat et al 2017).

Although hundreds of SNe are discovered each year by dedicated surveys (e.g., Law et al 2009, Leaman et al 2011, Holoien et al 2017), they are often too distant to resolve the SN ejecta and the immediate surroundings of the exploded stars

Laura A. Lopez

The Ohio State University, 140 W. 18th Ave., Columbus, OH 43210, USA

E-mail: lopez.513@osu.edu

Robert A. Fesen

Dartmouth College, 6127 Wilder Lab, Hanover, NH 03755, USA

E-mail: robert.a.fesen@dartmouth.edu 
(e.g., $1^{\prime \prime} \approx 50 \mathrm{pc}$ for a distance of $10 \mathrm{Mpc}$ ). While study of the closest SNe, such as SN 1987A (McCray and Fransson 2016), has advanced the field tremendously, our understanding of SN progenitors and the details of the explosion dynamics is severely hampered by the infrequency of nearby events.

Young, nearby supernova remnants (SNRs) offer the means to study SN explosions and dynamics at sub-pc scales, and thus they are crucial to test the predictions of SN models. Evolved SNRs are observable for up to $10^{5}$ years after the explosions across the electromagnetic spectrum, and multiwavelength campaigns have identified over 500 SNRs in the Milky Way and nearby galaxies (Badenes et al 2010, Sasaki et al 2012, Green 2014, Maggi et al 2016, Garofali et al |2017). Detailed investigations of SNRs that are a few thousand years old or less have yielded valuable insights into the progenitors, environments, and explosion dynamics for all SN subtypes (see e.g., Vink 2012 for a review).

Cassiopeia A (Cas A; $\simeq 23.4 \mathrm{kpc}$ ) is perhaps the most well-studied SNR in the Milky Way as it is the youngest known ( $\sim 350$ years old: Thorstensen et al 2001, Fesen et al 2006a) core-collapse SNR in the galaxy. Cas A resulted from an asymmetric Type IIb explosion (Krause et al 2008, Rest et al 2011) associated with a red supergiant progenitor with an initial mass of $15-25 M_{\odot}$ (Young et al 2006). The Chandra X-ray Observatory first-light image of Cas A revealed the neutron star produced in the SN (Pavlov et al 2000), and further X-ray investigations showed metal-rich ejecta primarily distributed in $a \approx 4^{\prime}$ shell (e.g., Hughes et al 2000, Hwang et al 2000, Willingale et al 2002). Kinematic studies of Cas A have mapped the three-dimensional structure of the ejecta (e.g., DeLaney et al 2010 , Grefenstette et al 2017), including the primary ring of SN debris as well as the high-velocity knots (up to $15,000 \mathrm{~km} \mathrm{~s}^{-1}$ ) that protrude beyond the shell in the northeast and southwest (Fesen and Gunderson 1996, Fesen 2001, Fesen et al 2006a, Fesen and Milisavljevic 2016).

The morphologies and kinematics of young SNRs like Cas A are important observables to test and constrain the SN explosion models being developed now. In this Chapter, we summarize the recent advances in studies of SNR morphologies $(\$ 2$ and SNR kinematics $(\$ 3$, particularly related to Cas A. We discuss how these results can be utilized to investigate the explosive origins of SNRs, the environments of SNRs, and the instabilities that mix ejecta with the surrounding medium.

\section{Morphologies of SNRs}

The morphologies of SNRs are complex and varied (see Figure 1). Consequently, significant effort has been made in terms of observations and interpretation of individual sources. However, through the decades, as more SNRs were imaged at radio, optical, and X-ray wavelengths, astronomers began to classify the sources based on their morphological characteristics (Mathewson et al 1983, Seward 1990, Williams et al 1999, Green 2014).

Remnant classifications used most commonly in the field (e.g., as in the Green Catalog of Galactic SNRs: Green 2014) are shell-type, center-filled, and composite. Shell-type SNRs are those whose emission is dominated by a limb-brightened shell (e.g., SN 1006: Winkler et al 2014). Center-filled SNRs have pulsar wind nebulae (PWNe; see review by Gaensler and Slane 2006) that shine brighter than their 

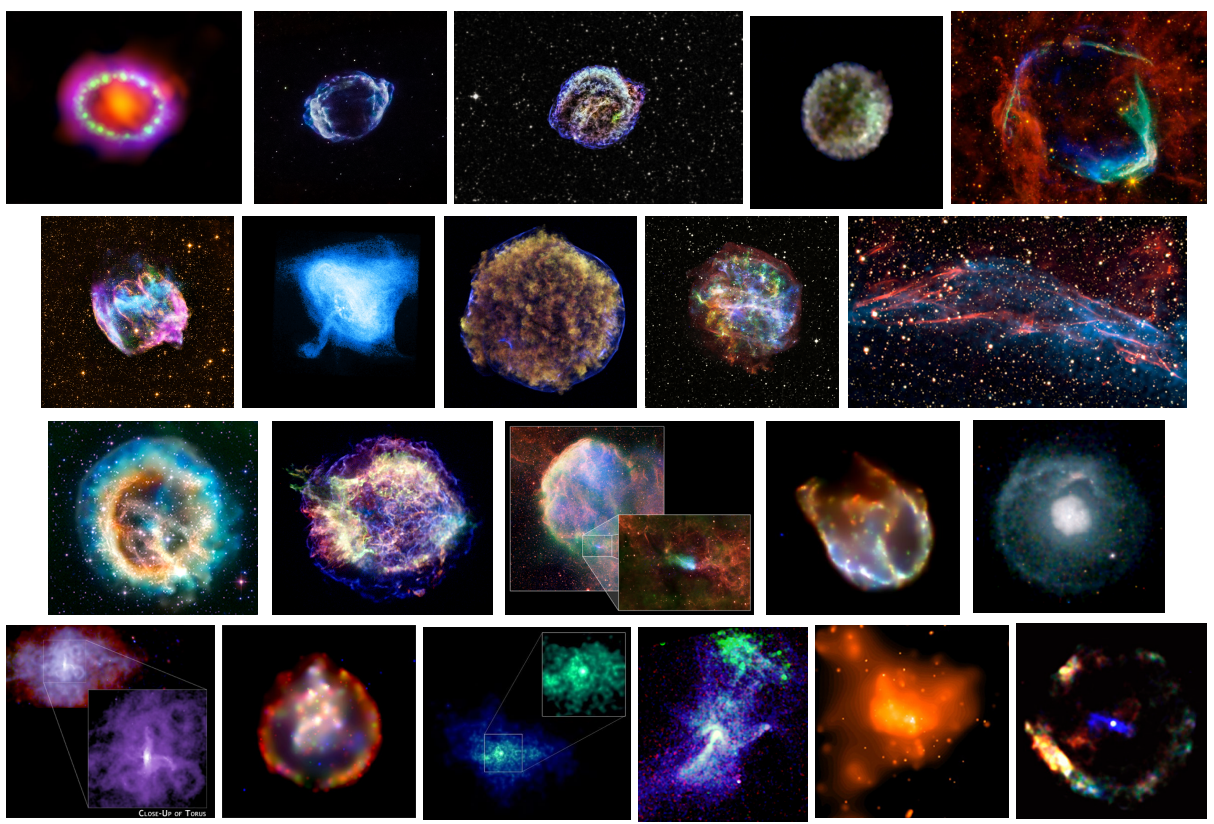

Fig. 1 Chandra X-ray Observatory images of a wide array of SNRs. Their morphologies are complex and heterogeneous, yet they are a rich sample from which to learn about explosions and their environments. These images were adapted from the Chandra press webpage (http://www.chandra.harvard.edu/press/).

shells (e.g., the Crab: Seward et al 2006). Composite SNRs are sources that have evidence of both a PWN and a shell in their radio and X-ray morphologies (e.g., MSH 11-56: Temim et al 2013). From X-ray imaging with Einstein and ROSAT, another class of SNRs was identified with radio shells and center-filled X-rays; these sources are called mixed-morphology or thermal-composite SNRs (Rho and Petre 1998, Shelton et al 1999).

\subsection{Effects of the Explosions on SNR Morphologies}

\subsubsection{Distinguishing Type Ia and Core-Collapse SNRs}

While SNRs were classified based typically on their radio and X-ray morphologies as described above, the relationship of those morphologies to the originating explosions (Type Ia or core-collapse) was unclear. The reason stemmed in part from the challenge of constraining the SN type of SNRs. Generally, SNe are classified phenomenologically based on their optical spectra and light curves near maximum brightness (Minkowski 1941, Filippenko 1997). Given that SNe are typed based on their properties days after an explosion, an alternative means is necessary to discern the explosions responsible for SNRs, which are hundreds to tens of thousands of years old.

The most reliable ways to classify SNR explosion types are the detection of a central neutron star (although chance alignments are possible: Kaspi 1998) or of 

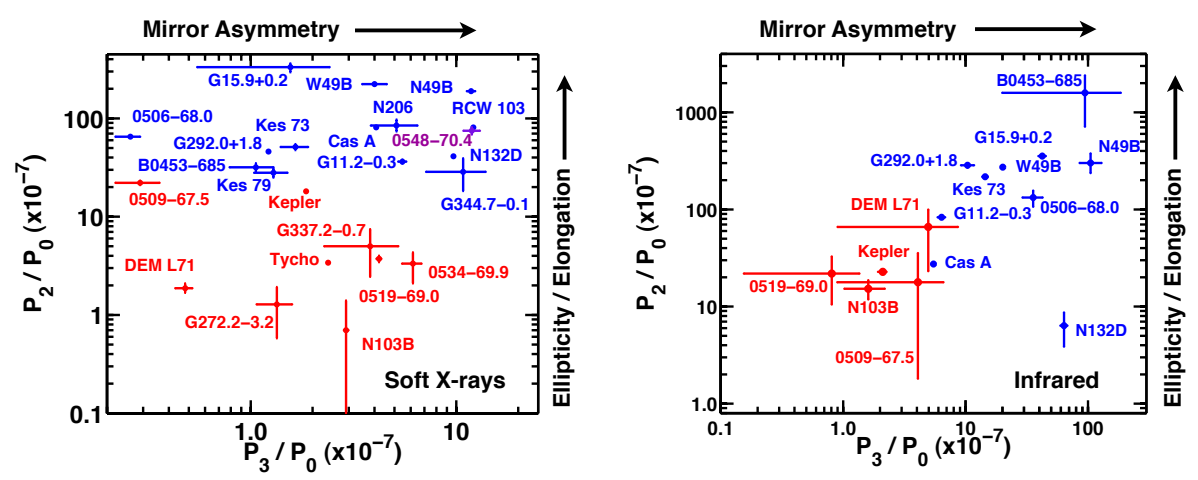

Fig. 2 Results from calculation of the multipole moments (a power-ratio method) to 24 Galactic and LMC SNRs: quadrupole power ratio $P_{2} / P_{0}$ (which measures ellipticity/elongation) vs. octupole power ratio $P_{3} / P_{0}$ (which quantifies mirror asymmetry) of the Chandra X-ray (left) and of the Spitzer $24 \mu \mathrm{m}$ (right) images. Type Ia SNRs are plotted in red points, and the CC SNRs are in blue (as classified by their abundance ratios, light echo spectra, neutron star detections, and/or their environments). The Type Ia SNRs separate naturally from the CC SNRs in both diagrams. Figures are adapted from Lopez et al (2011) and Peters et al (2013).

spectra from surrounding light echoes (e.g., Rest et al 2005, Krause et al 2008, Rest et al 2008). Another means to constrain the progenitor star is to measure the number of OB stars (e.g., Maggi et al 2016) or to investigate the stellar populations (e.g., Badenes et al 2009, Jennings et al 2012) in the vicinities of SNRs.

However, the most widely used method to type SNR explosions is to measure metal abundances using X-ray spectroscopy and compare the values to those predicted by $\mathrm{SN}$ models. In particular, the oxygen-to-iron ratio $(\mathrm{O} / \mathrm{Fe})$ is often employed for this purpose, since Type Ia SNe produce an order of magnitude more Fe than CC SNe, whereas CC SNe yield more intermediate-mass elements (e.g., $\mathrm{O}, \mathrm{Ne}, \mathrm{Mg}$ ) than Type Ia SNe (Woosley et al 2002). Using all of the approaches described above, many dozens of Milky Way and Magellanic Cloud SNRs have been identified as from Type Ia or from CC explosions.

Using a sample of young SNRs with well-constrained explosion types from the literature, Lopez et al (2009a) measured and compared the morphological asymmetries in the Si XIII Chandra X-ray images of Type Ia and CC SNRs using a multipole expansion technique called the power-ratio method (PRM; see Jeltema et al 2005 and Lopez et al 2009b for the mathematical formalism). These authors found that the Type Ia SNRs are more circular (have smaller quadrupole power ratios) and symmetric (have smaller octupole power ratios) than CC SNRs. In subsequent works, they extended the PRM to soft (0.5-2.1 keV) Chandra X-ray images and to Spitzer $24 \mu \mathrm{m}$ images (Lopez et al 2011, Peters et al 2013), and the results were consistent in those bandpasses as well (see Figure 2). These works suggest that the X-ray and infrared morphologies of SNRs may be used to constrain progenitors' explosions.

The SNR results described above are consistent with mounting evidence that Type Ia and CC SNe have intrinsically different symmetries. For example, spectropolarimetry studies - the measure of the polarization of light as a function of wavelength as it is scattered through the debris layers of expanding $\mathrm{SNe}$ - demonstrate that $\mathrm{CC}$ SNe have larger deviations from spherical symmetry than Type 
Ia SNe (e.g., Leonard et al 2006, Wang and Wheeler 2008, In some cases, the asymmetries of CC SNe can be substantial, altering the nucleosynthetic products of the explosions (e.g., Maeda and Nomoto 2003), ejecting metals at higher velocities (e.g., Mazzali et al 2007), and kicking newly-formed neutron stars to speeds $\lesssim 1000 \mathrm{~km} \mathrm{~s}^{-1}$ (Lyne and Lorimer 1994, Arzoumanian et al 2002, Hobbs et al 2005, Faucher-Giguère and Kaspi 2006).

It is worth noting that a systematic analysis of the Fe K-shell emission in Type Ia and CC SNRs recently showed that the Fe-K $\alpha$ centroid can also be used to type SNRs (Yamaguchi et al 2014). Specifically, these authors found that Type Ia SNRs have lower energy Fe-K $\alpha$ centroids than those of CC SNRs, indication that the Type Ia SNRs have less ionized plasmas than CC SNRs. This result indicates that Type Ia progenitors do not modify significantly their environments, and they reside in lower-density media than CC SNRs. Thus, although typing SNRs was a major challenge to the community twenty years ago, researchers now have a variety of tools to diagnose the explosive origins of SNRs.

\subsubsection{Connecting Neutron Star Kicks and SNR Morphologies}

Neutron stars are thought to possess a substantial "kick" velocity due to an asymmetric, CC explosion. Evidence for this is that NSs in the Milky Way are observed to have velocities of hundreds of $\mathrm{km} \mathrm{s}^{-1}$ (Lyne and Lorimer 1994, Arzoumanian et al 2002, Hobbs et al 2005, Faucher-Giguère and Kaspi 2006), up to $1000 \mathrm{~km}$ $\mathrm{s}^{-1}$ (e.g., Chatterjee et al 2005, Winkler and Petre 2007). Such large velocities are more than can be accounted for by the disruption of a close binary system (which produce velocities of $\sim 100 \mathrm{~km} \mathrm{~s}^{-1}$ : Lai 2001), suggesting an asymmetry in the dynamics of the explosion is the likely origin of NS kicks.

Two theories have been proposed to explain NS kicks. One scenario is that hydrodynamical instabilities lead to asymmetric mass ejection, accelerating the NS in a direction opposite to the bulk of ejecta (Scheck et al 2006, Wongwathanarat et al 2013b, Janka 2017). Such models predict that the heavier elements (e.g., Fe, Ti) are expelled directly opposite to the NS kick, whereas the intermediatemass elements (e.g., C, O, Ne, Mg) are only marginally affected. In another set of models, a NS kick arises from anisotropic neutrino emission. In this case, the neutrinos carry the bulk of the explosion energy, and both the NS and the ejecta are expelled in the opposite direction (Fryer and Kusenko 2006).

Recently, Holland-Ashford et al (2017) investigated the relationship between NS kick velocities and their associated SNRs' X-ray morphologies of 18 sources observed by Chandra and ROSAT. These authors found no correlation between the magnitude of asymmetries (i.e., the quadrupole and octupole power ratios) and the NS speeds (see Figure 3). However, they also showed that the SNRs' X-ray emission (as measured via the dipole angle of their $0.5-2.1 \mathrm{keV}$ images) was in the opposite direction as the NSs' motions in five out of six SNRs with well-constrained explosion sites (see Figure 4).

In addition, Holland-Ashford et al (2017) also noted that the X-ray emission of two of the SNRs (Cas A and G292.0+1.8) is dominated by the ejecta, and the other sources' morphologies are influenced at least in part by their complex environments. In a similar analysis to Holland-Ashford et al (2017), Katsuda et al (2017) showed that silicon, sulfur, argon, and calcium are distributed opposite to NS directions of motion in six young SNRs. The results of both works are consistent 

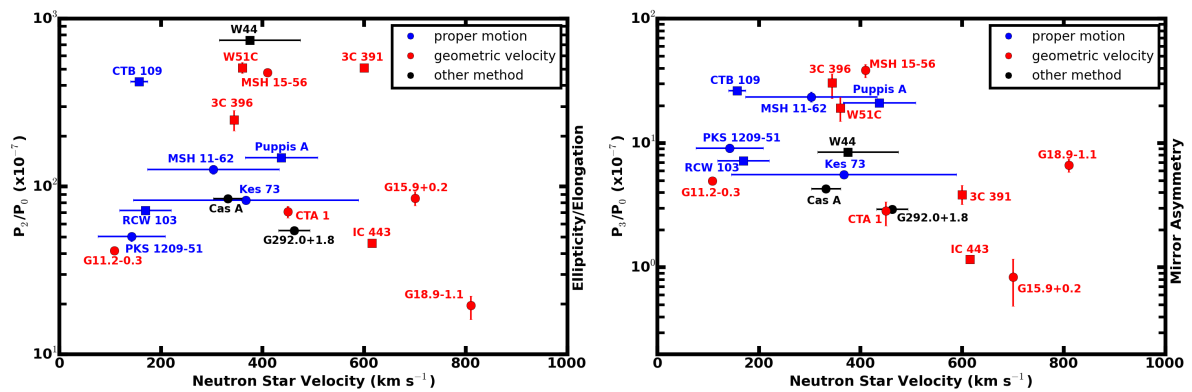

Fig. 3 The quadrupole (left) and octupole (right) power-ratios vs. neutron star velocities for a sample of 18 Galactic SNRs. Blue points are velocities measured via proper motions, and red points are velocities estimated from the spatial offset of the NS from the SNRs' geometric centers. Cas A and G292.0+1.8's NS velocities are determined by back-evolved filament motion to find the explosion site; W44's NS velocity is determined by multiple methods. Circles are used for SNRs without evidence of CSM/ISM interaction, and squares are those with clear evidence of CSM/ISM interaction. Figure is adapted from Holland-Ashford et al (2017).
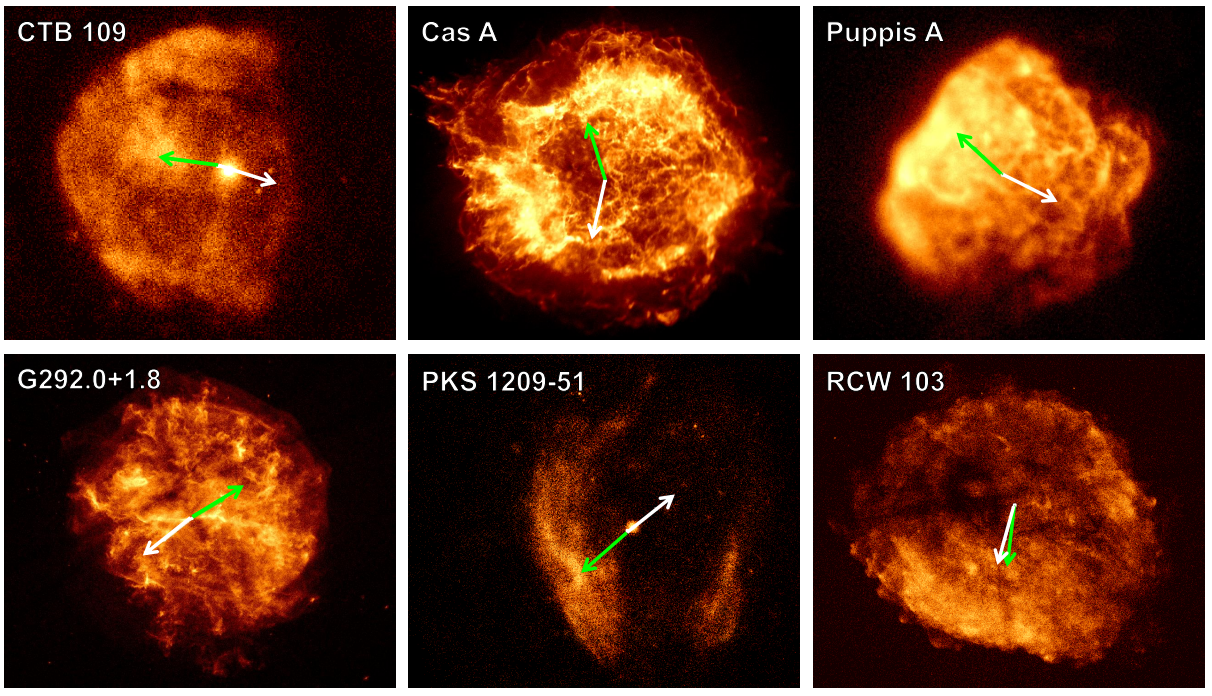

Fig. $4 \quad 0.5-2.1 \mathrm{keV}$ Chandra and ROSAT images of the six Galactic SNRs with robust measures of their explosion sites that are analyzed by Holland-Ashford et al (2017). The green arrow points from the explosion site to the direction of the dipole moment; the white arrow represents the direction of the NS motion. In Cas A and G292.0+1.8, the dipole moment direction reflects the bulk of ejecta emission. In CTB 109, Puppis A, and RCW 103, the dipole moment points towards enhanced emission due to interactions with CSM/ISM or a molecular cloud. It is unclear if the emission comes from ejecta or interactions in PKS 1209-51. Figure is adapted from Holland-Ashford et al (2017).

with predictions from models where NSs are kicked by ejecta asymmetries (Scheck et al 2006, Wongwathanarat et al 2013b, Janka 2017). 

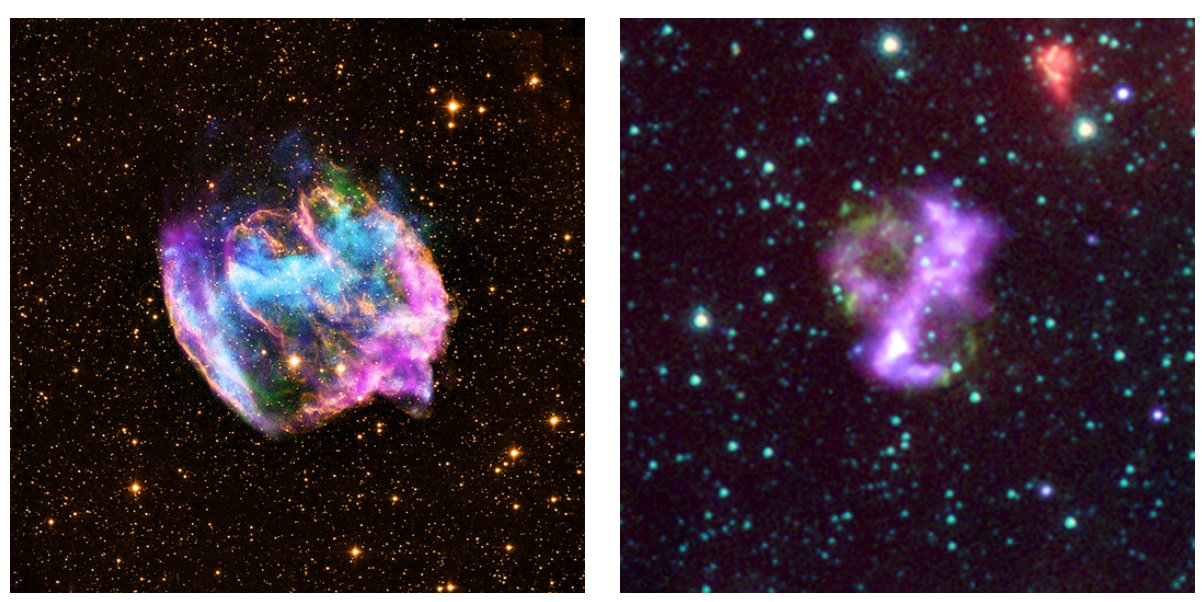

Fig. 5 Multiwavelength images of two extremely elliptical SNRs thought to be from jet-driven explosions (Lopez et al. 2013a, 2013b). Left: SNR W49B, with X-rays in blue, IR in yellow, and radio in purple. Right: SNR 0104-72.3, with X-rays in purple and IR in green. Images are from NASA/Chandra press releases.

\subsubsection{The Persistent, Elongated Morphologies of Jet-Driven SNRs}

The comparative, systematic analysis of SNR samples described in Sections 2.1.1 and 2.1 .2 is also an important means to identify and examine outlier sources. For example, two SNRs from the X-ray morphological studies have anomalously large elongations compared to other young remnants: W49B in the Milky Way and SNR 0104-72.3 in the SMC. Both are extremely elliptical (see the X-ray images in Figure 5, and the spatial distribution of iron in W49B is remarkable distinct compared to the other elements detected in X-rays (Lopez et al 2009b). Using spatially-resolved Chandra X-ray spectra, Lopez et al (2013b) and Lopez et al (2014) showed the mean abundance ratios in these SNRs are consistent with the predicted yields of jet-driven CC SNe. The identification of this kind of explosion in the SMC, where only 23 SNRs are known, may be evidence that jet-driven $\mathrm{SNe}$ occur more frequently in low-metallicity environments (since the SMC has a metallicity of $\sim 20 \%$ solar). In fact, recent work on long-duration gamma-ray bursts (which are thought to arise from jet-driven explosions) prefer low-metallicity environments (Modjaz et al 2008). Thus, it is possible that these two SNRs are local analogues to long GRBs observed as cosmological distances.

To test whether jet-driven explosions would retain their elliptical morphologies a few thousand of years after SN explosion, González-Casanova et al (2014) performed two-dimensional simulations of a jet-driven SN, following its subsequent evolution as a SNR. Figure 7 shows the synthetic thermal emission maps derived from this work. These authors performed two separate hydrodynamical calculations, each equipped to describe the behavior of the bipolar outflow at two different epochs. They used a simulation with a general equation of state and a reaction network to determine the nucleosynthesis that accompanies a jet propagating through a massive star (left panel). As demonstrated in the abundance distributions in Figure 7, the jetted outflow that is responsible for synthesizing 

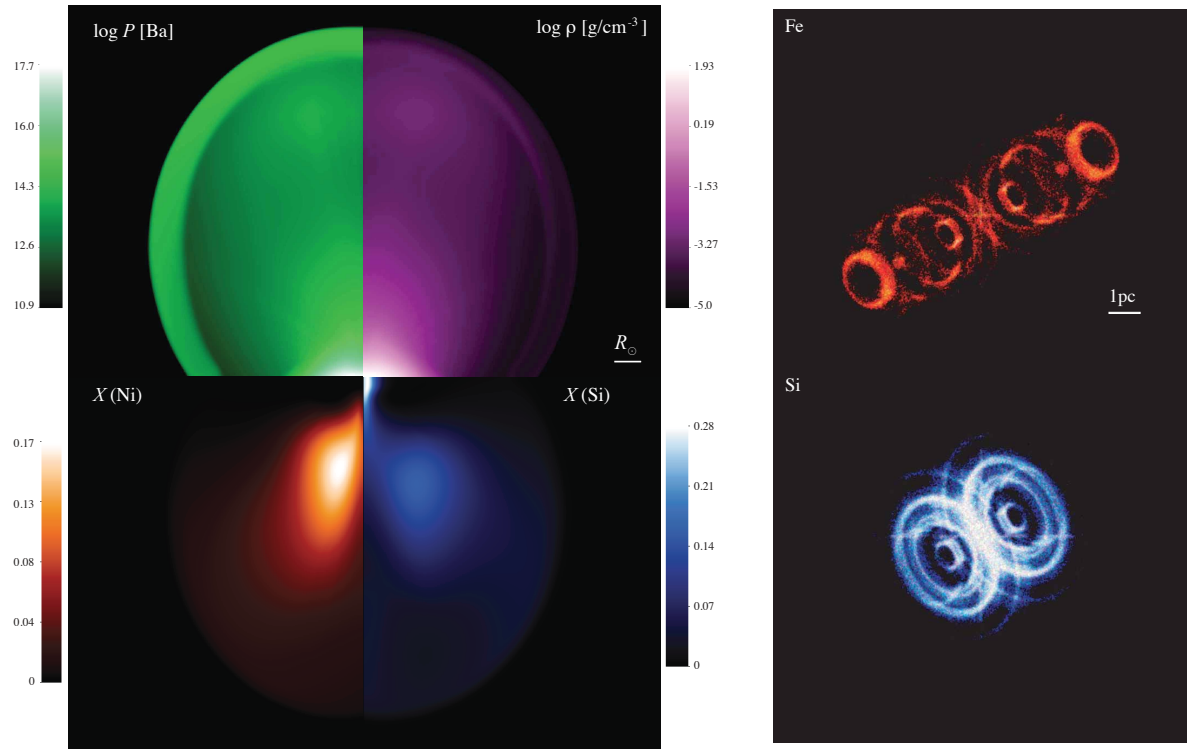

Fig. 6 The X-ray properties of a jet-driven SN remnant as seen in simulations. Left Panel: A jet-driven supernova remnant. it Left panel: Jet-driven SN explosion at the onset of the homologous phase, which is achieved 100 seconds after the bipolar ejecta has broken free from the $R_{*}=0.43 R_{\odot}$ stellar progenitor. The pressure $P$, mass density $\rho$ and the silicon $X(\mathrm{Si})$ and nickel $X(\mathrm{Ni})$ mass fractions are indicated in each frame with corresponding size scales in units of $R_{\odot}$. This model is then employed to calculate the evolution of a jet-driven SN remnant inside the wind bubble structure expected around a $25 M_{\odot}$ massive star for the case of an ISM pressure typical of the high-density molecular region surrounding the bipolar SNR. Right Panel: Fe XXV and Si XIII X-ray emission maps. The maps are obtained integrating the respective emission coefficients along the line of sight. The images are computed at an evolutionary age of $700 \mathrm{yr}$. To match the characteristics of W49B, the results of the simulation have been rotated $45^{\circ}$ with respect to the plane of the sky (in the direction of the observer), and $120^{\circ}$ with respect to the $z$-axis.

${ }^{56} \mathrm{Ni}$ carries more energy and inertia than the products of incomplete silicon and oxygen burning. Thus, the distribution asymmetry in the abundance is preserved as the SN material sweeps up the external medium.

The resulting outputs (i.e., the density, velocity, and compositional structure of the ejecta) were employed as the initial conditions in the calculation of the expansion and associated thermal X-ray emission produced by the hot shocked gas. The Fe XXV and Si XIII maps of the jet-driven SNR inside a wind bubble of a $25 M_{\text {sun }}$ star are shown in Figure 7 , right panel. Even in the presence of an uniform external medium, the X-ray morphology is dominated by the bright iron jet, with explosive oxygen-burning products (such as silicon and sulfur) enclosing the Fe, as observed in W49B. 
2.2 Influences of the Environment and the Surrounding Medium on SNR Morphologies

Since the progenitors of CC SNe have short main-sequence lives ( 3-50 Myr), their explosions are expected to occur within or near the dense media from which the massive stars formed. Consequently, it is common for CC SNRs to show traits of interaction with an inhomogeneous or dense CSM. For example, roughly one quarter of all Galactic SNRs show evidence of interaction with molecular clouds, such as the coincidence of $\mathrm{OH}$ masers (indicating the presence of shocked molecular gas: e.g., Wardle and Yusef-Zadeh 2002).

A SNR-molecular cloud interaction will have a profound influence on the Xray morphologies and spectra of SNRs. Large-scale density gradients can result in substantial deviations from spherical symmetry, and many SNRs interacting with molecular clouds have highly elliptical/elongated shapes compared to noninteracting CC SNRs (Lopez 2014, Holland-Ashford et al 2017). A large number of interacting SNRs are centrally bright with thermal X-ray emission, whereas their radio morphologies are shell-like (as described at the beginning of Section 2). Known as mixed-morphology (MM) SNRs, $\sim 40$ of these sources have been identified in the Milky Way (Vink 2012). Based on X-ray observations, MM SNRs can have enhanced metal abundances (Lazendic et al 2006) and/or isothermal plasmas across their interiors.

In young SNRs, shocks generate ionizing plasmas that slowly reach collisional ionization equilibrium (CIE). However, X-ray observations with $A S C A$ first revealed evidence that MM SNRs can have "overionized" plasmas (Kawasaki et al 2002 ,2005). In these cases, the electron temperature $k T_{\text {e }}$ derived from the bremsstrahlung continuum is systematically lower than the effective ionization temperature $k T_{\mathrm{z}}$ given by the line ratios. Subsequent Suzaku observations of MM SNRs detected radiative recombination continuum (RRC) features in their spectra (e.g., Yamaguchi et al 2009, Ozawa et al 2009, Uchida et al 2012), conclusive evidence of overionization.

In the collisional plasmas of SNRs, overionization is a signature of rapid electron cooling, and the physical origin of this cooling remains debated. One proposed scenario is thermal condution, where hot ejecta in a SNR interior cools by efficiently exchanging heat with exterior material (e.g., Cox et al 1999, Shelton et al 1999). Alternatively, the cooling may arise from adiabatic expansion, where the SN blast wave expands through dense CSM into a rarefied ISM (e.g., Itoh and Masai 1989, Moriya 2012, Shimizu et al 2012).

To ascertain which cooling scenario is responsible for overionization, the localization of the overionized plasma is crucial. Toward this end, Lopez et al (2013b) performed a spatially-resolved spectroscopic analysis to compare $k T_{\mathrm{e}}$ (derived by modeling the continuum) to $k T_{\mathrm{z}}$ (measured from the flux ratio of He-like to H-like lines). They found that the overionized plasma is concentrated in the west and has a gradient of increasing ionization from east to west. Given that the ejecta has is colliding with molecular material in the east and is expanding unimpeded in the west, the results suggest that adiabatic expansion is the dominant cooling mechanism of the hot plasma. 

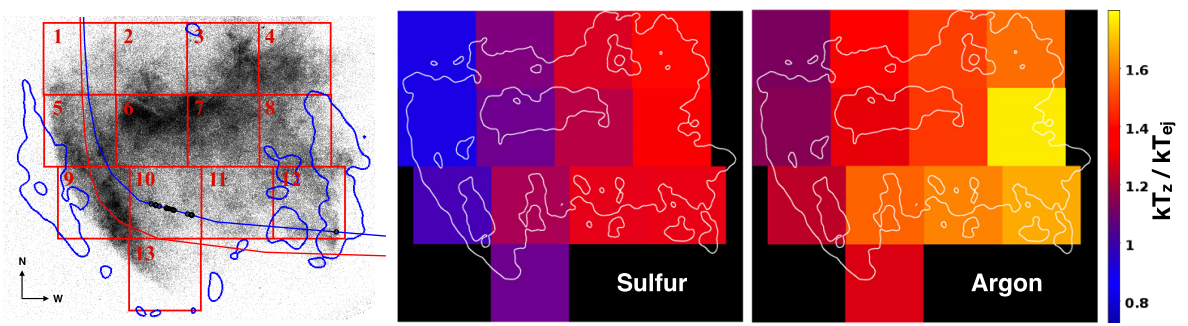

Fig. 7 Left: 13 regions analyzed by Lopez et al 2013a to map overionization in the SNR W49B. Regions are overplotted on the full-band $(0.5-8.0 \mathrm{keV})$ Chandra X-ray ACIS X-ray image, and the warm $H_{2}$ gas distribution is marked in blue The ejecta are colliding with the molecular gas in the east, whereas the ejecta are expanding freely in the west where the molecular material is thought to be in the foreground (Lacey et al 2001). Other panels: maps of overionization across W49B. Middle: ratio of ionization temperature $k T_{\mathrm{z}}$ (from the ratio of $\mathrm{S} \mathrm{XV}$ to $\mathrm{S} \mathrm{XVI}$ ) to the ejecta electron temperature $k T_{\mathrm{ej}}$ (from the bremsstrahlung continuum modeling). Right: maps of $k T_{\mathrm{z}} / k T_{\text {ej }}$ using the ratio of Ar XVII to Ar XVIII. Regions with $k T_{\mathrm{z}} / k T_{\mathrm{ej}}>1$ are overionized. Figures are adapted from Lopez et al (2013a).

\subsection{Instabilities \& Mixing}

On small scales, the distribution and scale of individual ejecta clumps can reveal the efficiency of the mixing between metals synthesized in the explosion and the surrounding CSM/ISM. Shock-heated ejecta expanding into lower-density material is subject to hydrodynamical instabilities (e.g., Rayleigh-Taylor instabilities) which are thought to be responsible for efficiently mixing ejecta metals (e.g., Blondin et al 2001. Wang and Chevalier 2001). X-ray and optical observations of individual SNRs show that these instabilities are capable of mixing ejecta out to and beyond the forward shock, in both Type Ia SNRs (e.g., in Tycho: Hwang et al 1998) and in CC SNRs (e.g., in Cassiopeia A: Fesen and Gunderson 1996, Hughes et al 2000, Fesen et al 2011).

Lopez et al (2011) investigated the mixing of ejecta in nine SNRs using narrowband Chandra images corresponding to emission lines from metals, including $\mathrm{O}$, $\mathrm{Ne}, \mathrm{Mg}, \mathrm{Si}$, and Fe. Specifically, they used wavelet-transform analysis to identify substructures and to measure their sizes. For example, Figure 8 displays the wavelet-transformed images of three emission line complexes ( $\mathrm{Mg}$ XI, Si XIII, and Fe XXv) in Cas A. They found that all nine sources have well-mixed ejecta: $90 \%$ of bright substructures of one element have corresponding substructures of the same size in another element, even if the metals arose from different burning processes. These findings reinforce observationally that hydrodynamical instabilities can efficiently mix ejecta in only a few hundred years.

\subsection{Bilateral SNRs and the Galactic Magnetic Field}

In the previous subsections, we have described how the thermal emission morphologies depend on the explosion and the environment. In addition, the morphology of the synchrotron radiation from SNR shocks gives clues about the surrounding medium, particularly the ambient density and the magnetic-field orientation. Specifically, Gaensler (1998) examined a sample of bilateral SNRs at radio fre- 

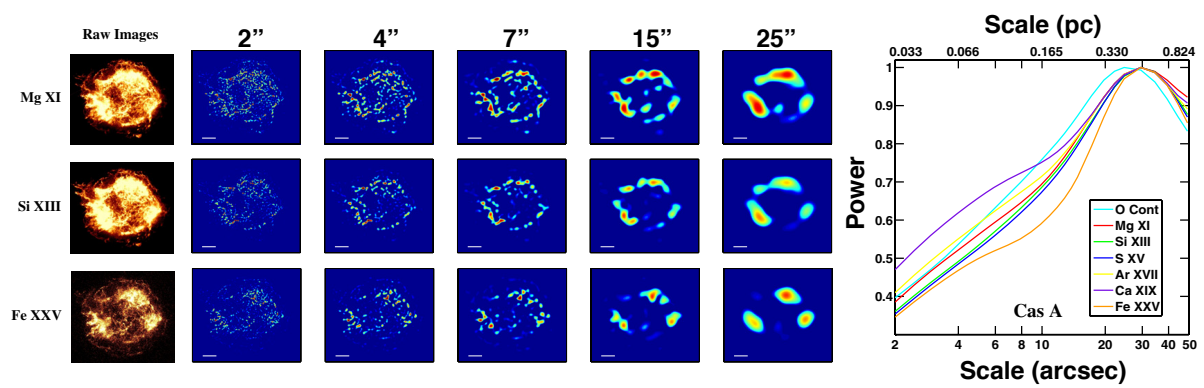

Fig. 8 Left panels: Wavelet-transformed images of Cas A showing the power at each scale for the Mg XI (top row), Si XIII (middle row), and Fe XXV (bottom row) as observed by Chandra. Right: Plot of the relative power as a function of scale for narrow-band images corresponding to emission lines detected in the Chandra observations of Cas A. The elements show similar profiles because they are well-mixed throughout the SNR, although some differences are evident (such as the Si XIII emission in the northeast jet and the Fe XxV located exterior to the Mg XI and Si XIII in the southeast). Figures are adapted from Lopez et al (2011).

quencies and showed that the bilateral axes tended to be aligned with the Galactic plane. However, some noteworthy exceptions existed, such as SN 1006, where the axis of symmetry is rotated almost $90^{\circ}$ from the Galactic plane.

Orlando et al (2007) performed three-dimensional magnetohydrodynamical (MHD) simulations of a spherically-symmetric shock propagating through a magnetized ISM. They found that a gradient in ambient density or in magnetic-field strength can produce asymmetric bilateral SNRs, as in e.g., objects with two radio limbs of different brightnesses.

Recently, West et al (2016) investigated the radio morphologies of all Galactic axisymmetric SNRs as well, including those with only one bright limb. They found that using a simple model of SNRs expanding into an ambient Galactic magnetic field, they were able to reproduce the observed radio morphologies of the sample. One implication of this result is that if the large-scale B-field is known near a source, then the distance to that SNR can be inferred. In subsequent work, West et al (2017) demonstrated that the synchrotron radio morphologies of their bilateral sample are more consistent with quasi-perpendicular cosmic-ray (CR) acceleration than with quasi-parallel case.

\section{Kinematics of Young SNRs}

As described in the previous section, the morphologies of SNRs are shaped in large part by their explosions but also by their environments. The other principal means to investigate the dynamics of the explosion mechanism and post-explosion expansion of SNRs is through kinematic studies of both radioactive and nonradioactive debris. In this regard, Cas $\mathrm{A}$ is a fortuitous, nearby target. As the youngest Galactic core-collapse SNR known (Fesen et al 2006b), it gives us a relatively high-resolution look at the expansion properties of the metals synthesized in the explosion.

Unless one views the Cas A remnant as peculiar and not representative of typical CC SN explosions, it can serve as a powerful and unique case to investigate 


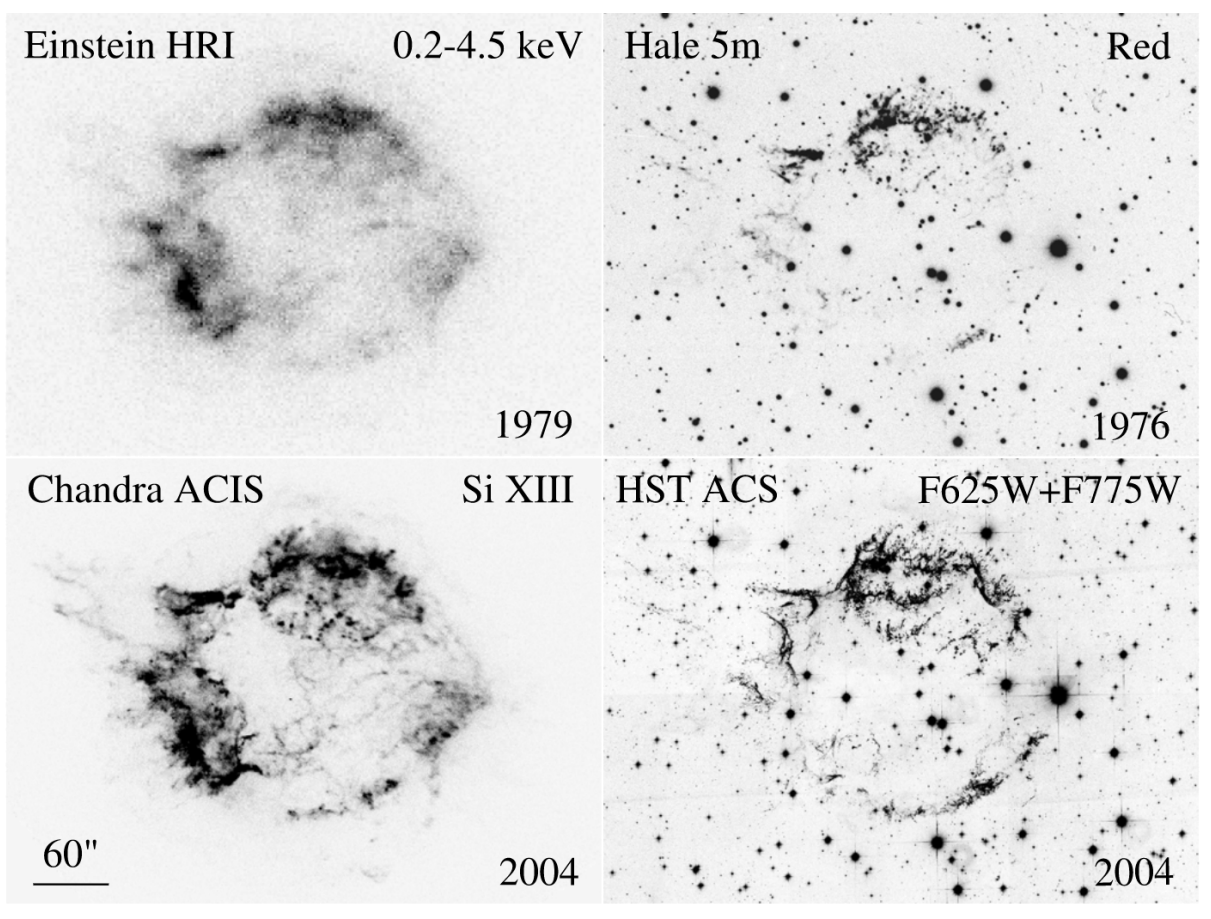

Fig. 9 Comparison of emission differences of Cas A as seen in X-rays and optical. Left hand panels show the 1979 Einstein X-ray image and a 2004 Chandra continuum-subtracted Si XIII $\mathrm{X}$-ray image. Right hand panels show broadband red optical images taken at similar epochs as the X-ray images. The optical images are sensitive to line emission from [S II] $\lambda \lambda 6716,6731$, [O I] $\lambda \lambda 6300,6364$, and [O II] $\lambda \lambda 7320,7330$. The Palomar Hale 5-m image is a 1976 July plate while the HST image is a co-add of 2004 ACS F625W and F775W filter images (Patnaude and Fesen 2014).

the kinetic properties of at least a subset and possibly a significant fraction of CC events. In this Section, we highlight the extensive work that has been done to characterize the kinematics of Cas A and what can be gleaned about the physics of explosions from this case study.

In terms of kinematics, Cas A is composed of three distinct sets of ejecta. The brightest at nearly all wavelengths is its thick main emission shell of ejecta. This material is shock-heated by the remnant's $\simeq 5000 \mathrm{~km} \mathrm{~s}^{-1}$ reverse shock and contains the bulk of Cas A's emitting debris. The shell's expansion is strongly asymmetrical with the rear hemisphere moving at roughly $6000 \mathrm{~km} \mathrm{~s}^{-1}$ while the front hemisphere is expanding around $2000 \mathrm{~km} \mathrm{~s}^{-1}$ slower. Surrounding this shell of shocked ejecta lie hundreds of small $\left(\leq 0.5^{\prime \prime}\right)$ ejecta knots with velocities between 7000 and $12,000 \mathrm{~km} \mathrm{~s}^{-1}$ with chemical compositions reflective of the outermost layers of the progenitor star at the time of explosion.

Cas A also exhibits two broad bipolar "jets" of especially high-velocity knots of ejecta $\left(\mathrm{v}=8000-15,000 \mathrm{~km} \mathrm{~s}^{-1}\right)$ along its NE and SW limbs. These broad plumes of debris consist of ejecta streams containing hundreds of small, individual ejecta knots. Interestingly, the fastest ejecta knots are richest in $\mathrm{S}, \mathrm{Ar}, \mathrm{Ca}$ - presumably from deep inside the progenitor - whereas ejecta knots near the jets' base are O- 
rich, suggesting an explosive origin pushing interior material up and out through the outer layers. It seems likely that the energy for the creation of these ejecta plumes/jets is somehow associated with the central explosion engine.

Chandra and Hubble Space Telescope (HST) images of Cas A taken over the last few decades have revealed a wealth of detail about its expanding main shell of S-, O-, Ar-, Ca-rich ejecta. However, it was obvious from the time of the first high-resolution X-ray image of Cas A taken with the Einstein satellite in 1979 that Cas A's X-ray and optical morphologies exhibit significant large-scale differences.

This is shown in Figure 9 which contrasts the remnant's X-ray and optical emissions in the late 1970s and in 2004, 25 years later. While there is some correlation between certain emission regions seen in the 1976 optical and 1979 X-ray images, especially along the remnant's northern limb, there is a poor large-scale optical - X-ray emission correlation over much of the remnant, especially in the south, east, and west quadrants. A better correlation is seen between the X-ray and optical emission in the 2004 Chandra and HST images.

Such comparisons make it clear that while Cas A's overall X-ray emission has undergone only minor large-scale changes over the last few decades, its optical appearance has gone from one of a faint and sparse emission structure to a bright and extensive emission morphology (Patnaude and Fesen 2014). Differences between $\mathrm{X}$-ray and optical images appear likely to be due to differences in the density of the reverse-shocked material; that is, X-ray emission regions highlight low density, interclump regions, whereas optical emission features are associated with much smaller, denser debris knots.

Although the remnant's X-ray and optical emissions in Cas A's main shell have grossly similar morphologies in certain regions, they possess different expansion velocity ranges and very different detailed structure. Kinematic differences between relatively cool and dense optical/infrared emitting knots and much hotter and more diffuse X-ray emitting debris can be seen in Figure 10. Such expansion velocity differences are discussed in more detail below in Section 3.1 .

Outside of the main shell of reverse-shocked material, HST images have uncovered nearly 4,000 outlying fragments around the remnant and largely out ahead of the $4500-5000 \mathrm{~km} \mathrm{~s}^{-1}$ blast wave. These knots exhibit proper motions indicating transverse velocities of up to $12,000 \mathrm{~km} \mathrm{~s}^{-1}$ (Fesen 2001, Fesen et al 2006b). They surround nearly all regions about the main shell and are distinctly different in both chemistry and expansion velocity than the remnant's NE and SW jets. Most of these knots are too small and faint to detect from the ground. The expansion and chemical properties of these outer ejecta knots is shown in Figure 11.

As mentioned above, the presence of weak hydrogen emission lines in the spectra of these outer knots (which indicate that the Cas A progenitor had some photospheric hydrogen at the time of outburst) is consistent with the SN IIb classification based on light echo studies. Faint streams of trailing emission indicate ablated material off these outer SN fragments as they move through the local ISM/CSM environment have been detected in deep HST images, showcasing the earliest stage of ISM enrichment by SN debris. 

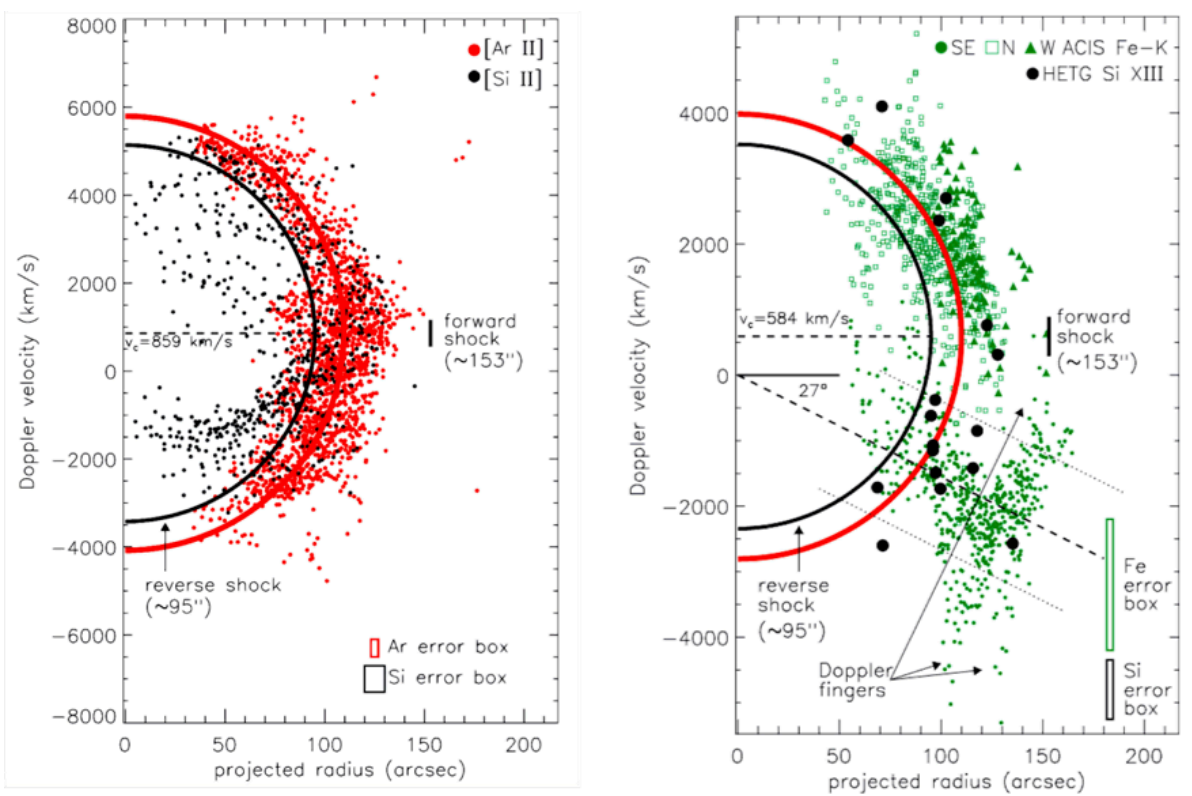

Fig. 10 Left panel: Doppler velocity vs. projected radius for infrared [Ar II] emissions in red and $[\mathrm{Si}$ II] in gray. The mean velocity errors and spatial resolution are indicated by error boxes at the bottom of the figure. The red semicircle represents the best-fit spherical expansion model. The black semicircle represents the reverse shock and the projected radius of the forward shock is also indicated. Right panel: Doppler velocity vs. projected radius for X-ray Fe-K emissions in green and Si XIII emissions in black. Different symbols are used to represent the north (open square), west (filled triangle), and southeast (filled circle) Fe-K distributions (DeLaney et al 2010

\subsection{Ejecta Rings and Interior Bubbles}

Unlike the more or less random arrangement of debris expelled by a supernova explosion one might have expected, the kinematic and chemical properties of Cas A's main shell of ejecta has been found to consist of a series of large optical and infrared rings of reverse shocked O-, S-, Ar-, Ca-rich ejecta. These rings surround much of the remnant's X-ray emitting Fe-rich ejecta. This unexpected chemical arrangement of ejecta can be seen in Figures 12 and 13 where the remnant's main shell ejecta can be seen to lie almost exclusively in ring-like structures.

Reed et al (1995) and Lawrence et al (1995) were the first to establish the existence of conspicuous rings of reverse-shocked ejecta in Cas A. More recently, DeLaney et al (2010) and Milisavljevic and Fesen (2013) identified about half a dozen well-defined ring-like structures with angular diameters between $30^{\prime \prime}$ and $120^{\prime \prime}(0.5-2 \mathrm{pc})$. Most of these rings are actually short cylinders giving them a crown-like appearance in $3 \mathrm{D}$ projections.

The height in velocity space of these crowns radially away from the remnant's center is up to $1000 \mathrm{~km} \mathrm{~s}^{-1}$. This radial extent may be related to the $\simeq 20-30$ year radiative timescale for cooling of these reverse-shocked ejecta knots.

The presence of these ejecta rings naturally led to questions about their nature. DeLaney et al (2010) noted the coincidence of large ejecta rings with the three 


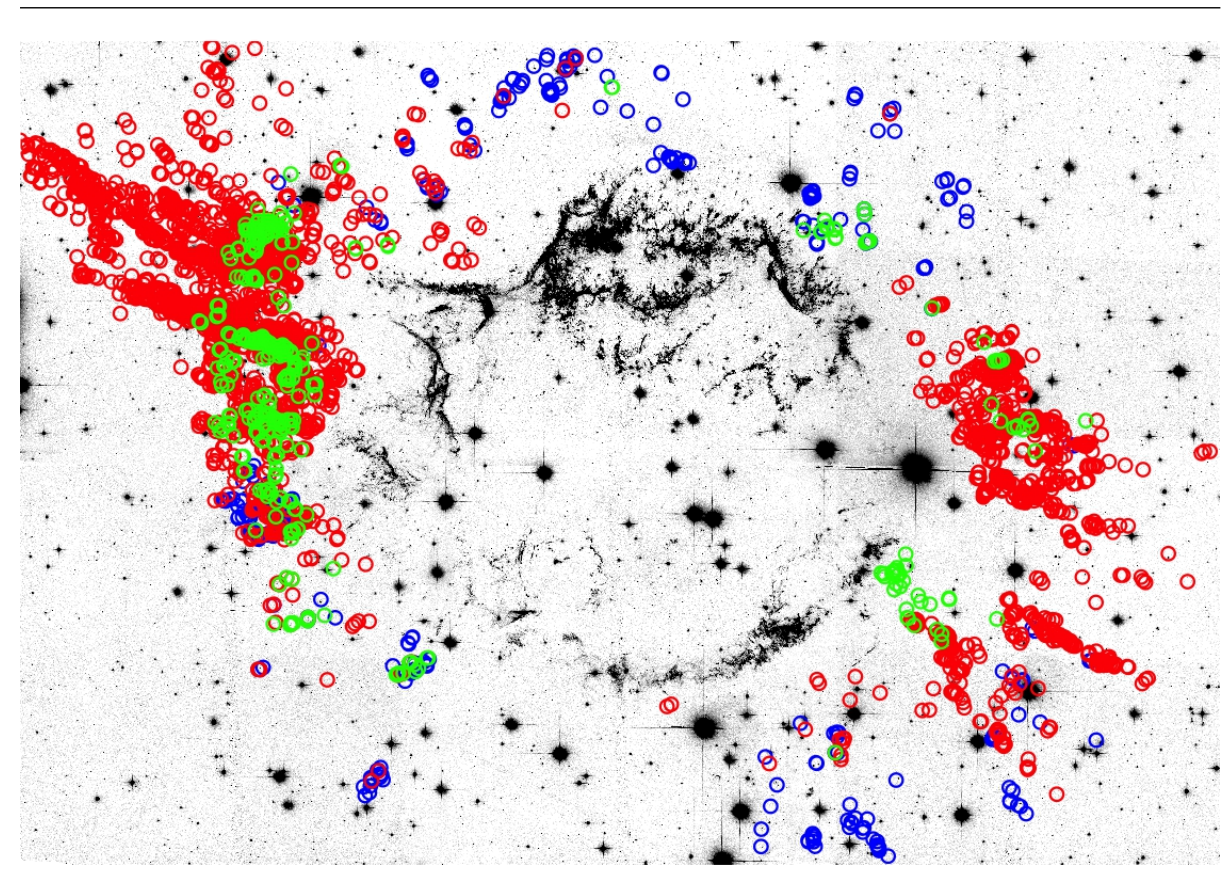

Fig. 11 Locations of high-velocity ejecta outside of the remnant's main shell of ejecta shown on a 2004 HST image. Blue and green circles indicate N and O emission knots while red circles mark S emission knots (Fesen and Milisavljevic 2016).

regions of Fe-K X-ray emission and suggested that these and other, less prominent features are regions where the ejecta have emerged from the explosion as "pistons" of faster-than-average ejecta. In this view, the remnant's main shell rings represent the intersection points of these pistons with the reverse shock, similar to the bowshock structures.

An alternative explanation first suggested by Blondin et al $(2001)$ is that the observed ejecta rings represent cross-sections of large cavities in the expanding ejecta created by a post-explosion input of energy from plumes of radioactive ${ }^{56}$ Ni-rich ejecta. From Spitzer infrared and Chandra images and spectra, DeLaney et al (2010) were able to show that Fe-rich X-ray emitting material was surprisingly confined inside the remant's optical/IR emitting ejecta rings. This structure can be seen in Figure 13 where the Fe-rich ejecta (blue) is found inside rings of S-, O-rich ejecta (red).

Turbulent motions that would initiate this Ni bubble structure in Cas A are not unlike recent 3D simulations of the large-scale mixing that takes place in the shockheated stellar layers ejected in the explosion of a $15.5 M_{\odot}$ blue supergiant star presented in Hammer et al (2010). As shown in their models, a progenitor's metalrich core is partially turned over with nickel-dominated fingers overtaking oxygenrich bullets. Although the evolution of these simulations is strongly dependent on the internal structure of the progenitor star (Ugliano et al 2012), it is still tempting to draw an association between the Ni-rich outflows seen in the Hammer et al (2010) models and the rings of Cas A. 

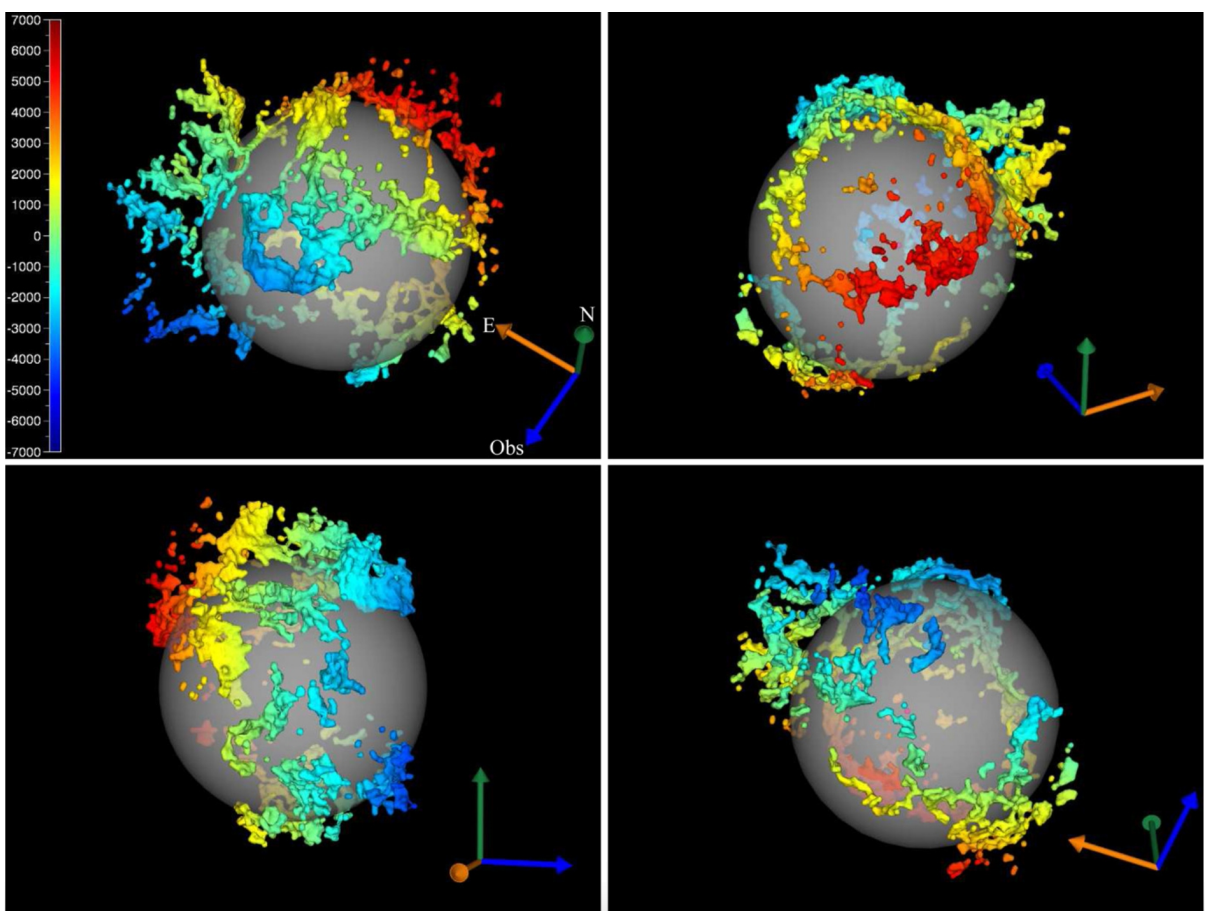

Fig. 12 Three-dimensional projections of the optical emission from Cas A's main shell illustrating the ubiquity of large ejecta rings. Noticeable rings of ejecta include the blueshifted ring in the north and the much larger neighboring redshifted ring (Milisavljevic and Fesen 2013).

Discovery of Cas A's ejecta rings was followed-up by the detection of unshocked material inside the remnant (Isensee et al 2010, Milisavljevic and Fesen 2015). Cas A's interior material consists of a handful of large cavities or bubbles, likely the result of plumes of radioactive ${ }^{56} \mathrm{Ni}$ which expand, pushing non-radioactive material like O, S, Ar, and Ca into bubble-like structures. Such an arrangement explains the observed Fe-rich ejecta inside rings of O-, S-rich debris. When the expanding bubbles encounter the remnant's reverse shock, the intersection is in the form of rings filled with Fe-rich ejecta, the product of ${ }^{56} \mathrm{Ni}$ decay.

Consequently, it now appears that the rings of ejecta seen nearly everywhere in Cas A are a product of being the cross-section of large, internal cavities when they encounter the inward moving reverse shock front. Similar rings of ejecta to those seen in Cas A, thought to be due to an interior bubble-like structure, are present in other CC SNRs.

Figure 14 shows a comparison of Mercator maps of emitting ejecta for Cas A (upper planel) and for the much lower expansion velocity and Crab-like SNR 3C 58 (lower panel). In both cases, only a handful of rings - not dozens - are present in these two CCs SNRs despite the difference in their estimated progenitor masses: 15-20 $M_{\odot}$ for Cas A (Young et al 2006) and 8-10 $M_{\odot}$ for 3C 58.

The presence of large-scale ejecta rings in CC SNe may help explain the clumpy emission-line profiles seen in late-type spectra of SNe II/Ib events. Milisavljevic et al (2012) showed how Cas A's main shell spectra would appear as an unre- 

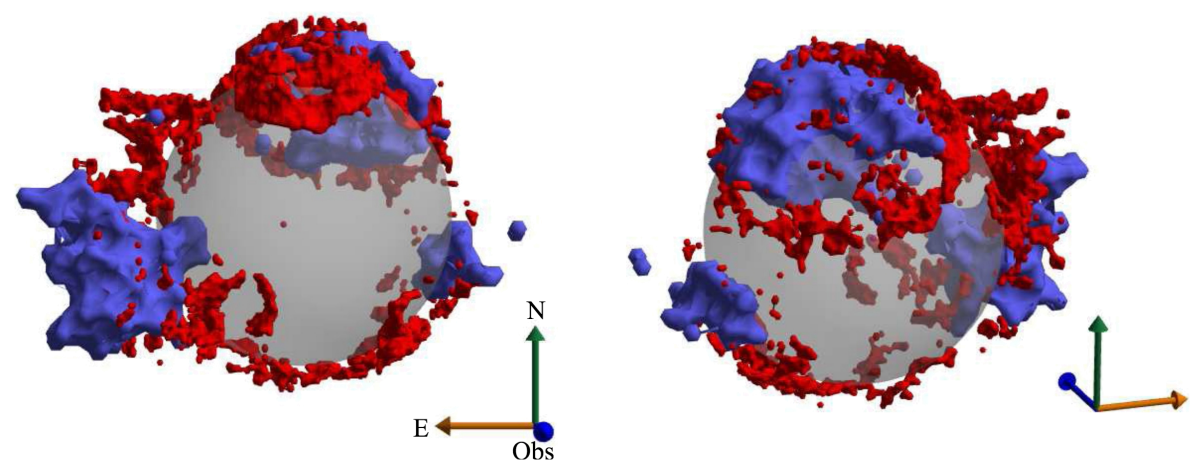

Fig. 13 Location of iron-rich X-ray emitting ejecta (blue) with respect to the main shell sulfurand oxygen-rich optically emitting ejecta (red). The X-ray data shown are from DeLaney et al. (2010). Note in the right-hand panel the location of the iron-rich (blue) ejecta well confined inside the large red ring of S,O-rich ejecta. Adapted from Milisavljevic and Fesen (2013).

solved extragalactic source. Similarities were seen between the integrated Cas A spectrum and several late-time optical spectra of decades-old extragalactic SNe. Particularly well matched with Cas A were SN 1979C, SN 1993J, SN 1980K, and the ultraluminous SNR in NGC 4449.

Since the emission-line substructure observed in the oxygen forbidden line profiles of Cas A is associated with the large-scale rings of ejecta, Milisavljevic et al (2012) suggested that similar features in intermediate-aged SNe (which have often been interpreted as ejecta "clumps" or "blobs") are, in fact, probable signs that large-scale rings of ejecta are common in these sources.

In summary, kinematic structures in $\mathrm{CC}$ SNe are highly dependent on the elemental abundances of the ejecta. This is apparent in Cas A's main shell where Fe-rich ejecta is largely confined to certain regions surrounded by nonradioactive SN debris. It is also true regarding the higher-velocity ejecta knots around the remnant and out ahead of the forward shock. These ejecta knots are the only ones which contain any hydrogen, a signature that the progenitor SN had a thin layer of hydrogen, leading to a SN II spectrum at early epochs. Lastly, Cas A's very high-velocity ejecta jets display a strong radial 'upside-down' chemistry unlike anywhere else in the remnant.

\subsection{High-Velocity Ejecta "Jets"}

It is of interest to consider the presence of jet-like ejecta streams seen in Cas A in the context of recent work on supernovae that has established a continuum of explosion energies extending from broad-lined SNe Ic associated with gamma-ray bursts to more ordinary SNe Ibc (Soderberg et al 2010, Chakraborti et al 2015). The existence of jets across such explosive events suggests that a wide variety of jet activity may potentially be occurring at energies that are hidden observationally.

The nature of the anomalously high velocities of ejecta in Cas A's NE and SW regions has long been a puzzle. Asymmetric debris structures produced by circumstellar interaction have been theoretically modeled by Blondin et al (1996), 

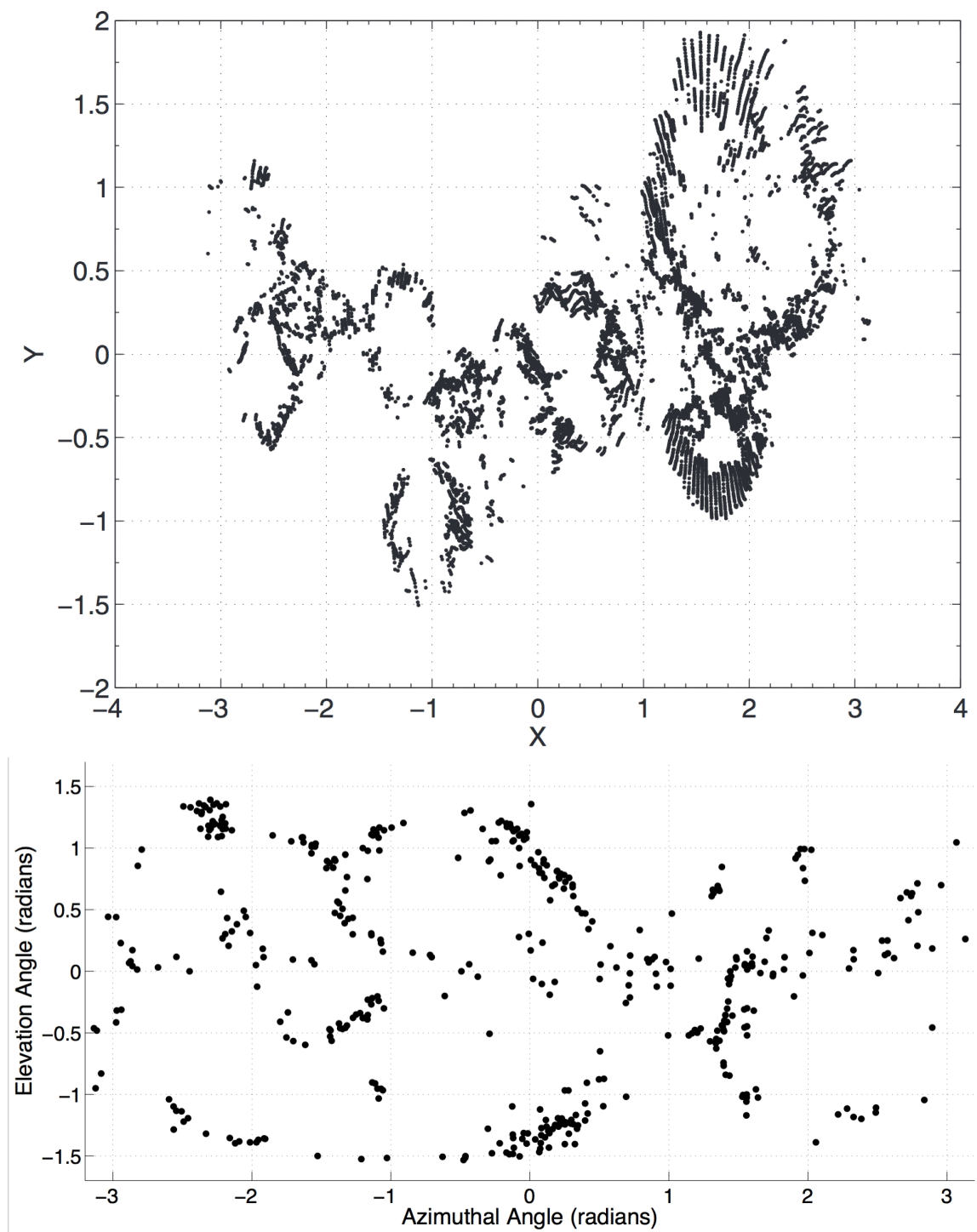

Fig. 14 Mercator projections of ejecta in Cas A and 3C 58. Upper Panel: The main shell of Cas A's optically emitting ejecta (Milisavljevic and Fesen 2013). Lower Panel: High-velocity emission knots in the Crab-like SNR 3C 58 (Fesen et al. in prep.). In both projections, the linear scale is equal in all directions around any point and conformal, but the cylindrical map projection distorts the size and shape of large objects, especially toward the poles.

who showed that a jet-like feature of SN ejecta can be generated in the progenitor's equatorial plane from pole/equator density gradients in the local CSM. Thus, these high-velocity regions could be secondary features caused by instability-powered flows from an equatorial torus, where the explosion axis is loosely defined by the X-ray iron-rich regions found in the SE and NW. 
However, the very prominent rupture-like features in Cas A's main shell near the base of the $\mathrm{NE}$ jet is visible in X-rays, optical, IR, and radio images and are certainly suggestive of an explosive formation process. Although perhaps not indicative of a jet-induced explosion, there is nonetheless substantial evidence that the NE and SW jets are associated with core-collapse explosion dynamics in some way.
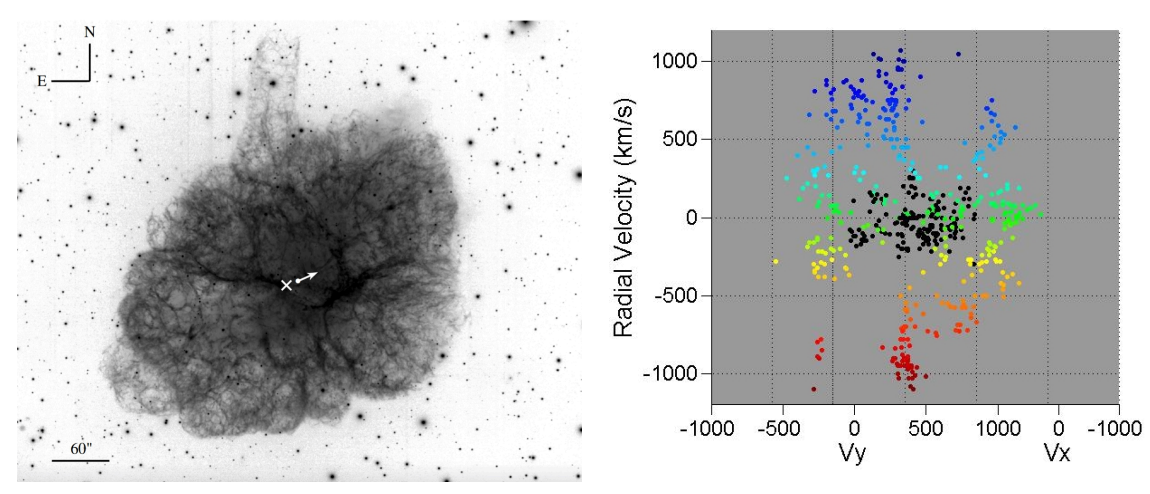

Fig. 15 Left Panel: An [O III $] \lambda \lambda 4959,5007$ image of the Crab Nebula showing its northern jet with respect to the entire remnant. The ' $\mathrm{X}$ ' marks the Nugent (1998) centre of expansion, and the white dot and arrow marks the pulsar and its proper motion direction as determined by Kaplan et al (2008). Right Panel: 2D projection of observed radial velocities (color-coded) vs. transverse RA and Dec velocities for optical emission knots in the young Crab-like SNR 3C 58 assuming an explosion age of 2200 years. Slower moving CSM knots are shown in black. Note that the wide north and south openings (jets) exhibit a similar broad opening morphology to that seen in the Crab's northern filamentary jet feature (Fesen et. al. in prep.).

The location of chemically distinct, optically-emitting knots in both jets is also consistent with an unusual, high-velocity ejection of underlying mantle material (Fesen 2001). Knots exhibiting a mix of $\mathrm{H} \alpha,[\mathrm{N} \mathrm{II}],[\mathrm{O} \mathrm{II}],[\mathrm{S} \mathrm{II}]$, and [Ar III] line emissions are only observed in the two jet regions, consistent with an eruptive and turbulent mixing of the underlying S-, O-, and Ar-rich material with photospheric H- and N-rich layers (Fesen and Gunderson 1996, Fesen 2001). Furthermore, spectra of optical knots in the NE and SW jets lying farthest out and possessing the highest ejection velocities show no detectable emission lines other than those of [S II] and [Ca II], suggesting an origin from the Si-, S-, Ar-, Ca-rich layer deep inside the progenitor star.

Kinetic energy estimates for the Cas A jets are around $1 \times 10^{50}$ erg (Fesen and Milisavljevic 2016). An explosive origin of the jets would mean this value may be an underestimate since it does not include the energy needed to propel the material in both jets up through Cas A's progenitor's outer layers. This added energy, together with the possibility of additional mass from undetected diffuse jet material, means the jets likely contain $10 \%$ or more of the expected $10^{51}$ erg SN explosion energy.

Cas A is not alone in being a young CC SNR with jet-like structures (see e.g., Section 2.1.3). As shown in Figure 15, low-mass progenitor CC SNe, such as the Crab Nebula and 3C 58, exhibit broad, plume-like ejecta streams. The fact that jet-like streams of unusually high-velocity material are present in both low- 
mass progenitors like the Crab and high-mass progenitors as in GRBs may be an indication that the central engine dynamics are not dis-similiar.

One additional clue as to the nature of Cas A's NE and SW ejecta jets and the explosion dynamics comes from examining the kinematic properties of the inferred motion of the remnant's neutron star. Its location some $7^{\prime}$ south of Cas A's expansion center (see Figure 4, suggests a transverse velocity "kick" of $350 \mathrm{~km} \mathrm{~s}^{-1}$ imparted during the explosion (Fesen et al 2006a). Intriguingly, the projected line connecting the NE and SW jets lies nearly perpendicular to the inferred direction of the NS (see Figures 4 and 11). Assuming the NE/SW axis to be the most significant in the original core-collapse, this runs counter to most jet-induced explosion models that predict that the neutron star will undergo a kick roughly aligned with the jet axis.

\subsection{The Kinematics of Radioactive Titanium in Young SNRs}

With the launch of $N u S T A R$, the first focusing, hard X-ray (3-79 keV) observatory (Harrison et al 2013), it is possible to measure the kinematics of ${ }^{44} \mathrm{Ti}$ from the radioactive decay lines at 68 and $78 \mathrm{keV}$. To date, these lines have been detected in two young CC SNRs: SN 1987A (Boggs et al 2015) and Cas A (Grefenstette et al 2014, 2017). In the case of SN 1987A, the lines were narrow and redshifted with a velocity of $\sim 700 \mathrm{~km} \mathrm{~s}^{-1}$, and the lack of a blueshifted component suggests large-scale asymmetry of the ejecta.

In Cas $\mathrm{A}$, the ${ }^{44} \mathrm{Ti}$ was localized using the $N u S T A R$ observations, and one major surprise was that the ${ }^{44} \mathrm{Ti}$ did not trace the distribution of Fe K (see Figure 16 , left panel), nor does it appear associated with the NE/SW jets (Grefenstette et al 2014). Using deeper NuSTAR data, Grefenstette et al (2017) performed spatiallyresolved spectroscopic analysis of the ${ }^{44} \mathrm{Ti}$ ejecta and found that $\sim 40 \%$ of the material is interior to the reverse shock, $40 \%$ is at/near the reverse shock, and $20 \%$ is beyond the reverse shock (see Figure 16 , right panel). Generally, the ${ }^{44} \mathrm{Ti}$ interior to the RS is not correlated with observed features in the optical or infrared. The ${ }^{44} \mathrm{Ti}$ exterior to the RS is co-located with the shock-heated Fe, whereas regions with $\mathrm{Fe}$ do not necessarily have associated ${ }^{44} \mathrm{Ti}$. The authors interpreted this result as evidence that local conditions during the explosive nucleosynthesis varied enough to suppress the production of ${ }^{44} \mathrm{Ti}$ in some regions. Based on the ${ }^{44} \mathrm{Ti}$ line centroids, the majority of the material is receding along the line of sight, with velocities ranging from $1,100-5,700 \mathrm{~km} \mathrm{~s}^{-1}$ (though there is some blueshifted emission as well). The flux-weighted average of the ${ }^{44} \mathrm{Ti}$ velocities gives the direction of this ejecta component that is almost exactly opposite to the direction of the Cas A NS, similar to the results presented in Figure 4

\section{Conclusions and Future Prospects}

The morphology and kinematics of SNRs are an especially powerful means to test and constrain SN explosion models. The predictive power of simulations has improved dramatically over the last few years, and the results can be compared to observational characteristics of SNRs, including large-scale compositional and morphological asymmetries as well as the three-dimensional kinematics of ejecta 

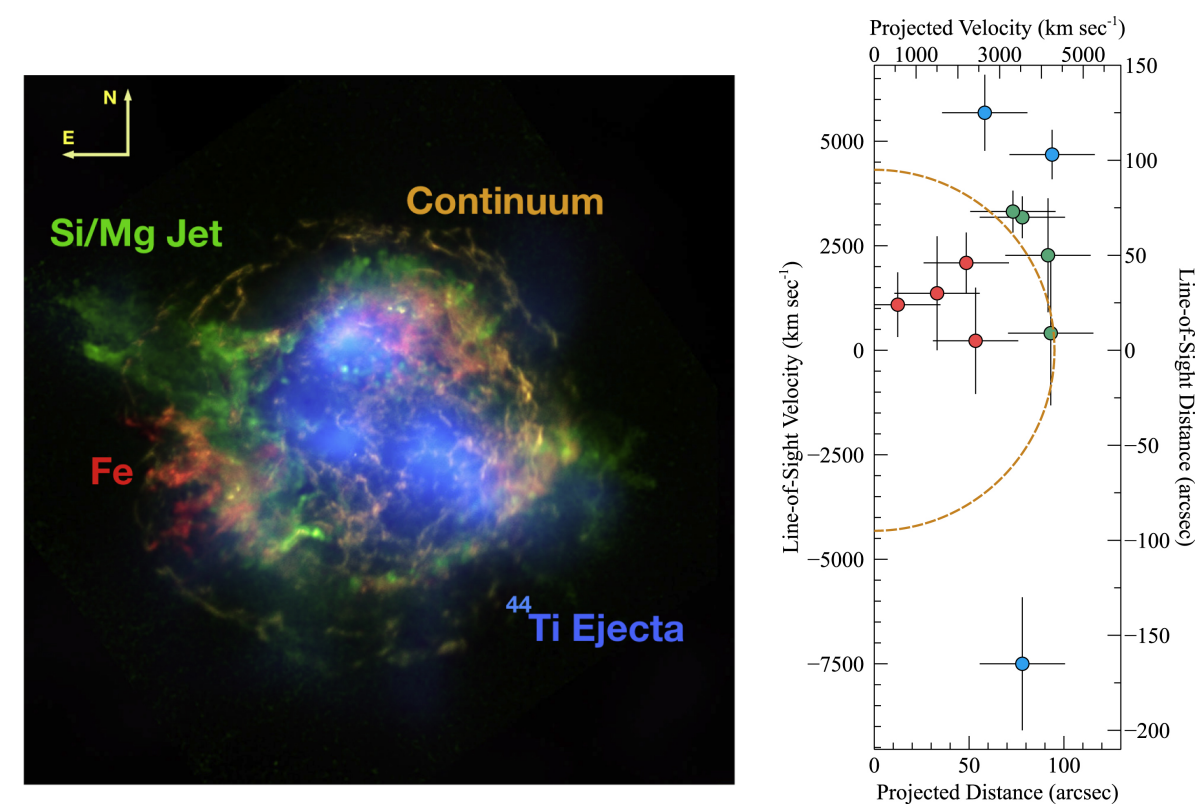

Fig. 16 Left: Three-color image of Cas A, with ${ }^{44} \mathrm{Ti}$ (from NuSTAR observations) in blue, $4-6$ $\mathrm{keV}$ continuum in gold, Fe in red, and $\mathrm{Si} / \mathrm{Mg}$ in green (the latter from Chandra observations). Right: The light-of-sight velocity of the detected ${ }^{44} \mathrm{Ti}$ versus the projected distance from the center of expansion of Cas A, where positive velocities indicate redshift. The gold dashed circle represents the location of the reverse shock; thus, the red points are interior to the RS, green points are at/near the RS, and blue points are exterior to the RS. Figures are from Grefenstette et al (2017).

material. In particular, as the youngest known CC SNR in the Milky Way, Cas A offers an up-close view of the complexity of these explosive events that are unresolved in extragalactic sources.

In the future, an exciting prospect in the study of SNRs is X-ray microcalorimeters. CCD energy resolution is insufficient to resolve He-like and H-like line complexes of ions at X-ray wavelengths, and grating spectrometers are only useful when objects have minimal angular extension (e.g., SN 1987A: Dewey et al 2008. SNR 1E 0102.2-7219: Flanagan et al 2004) or if the ejecta knots are sufficiently bright and isolated (e.g., Cas A: Lazendic et al 2006, G292.0+1.8: Bhalerao et al 2015). X-ray microcalorimeters are non-dispersive spectrometers that can take spatially resolved, high-resolution (with a few eV resolution) spectra across extended objects, like SNRs and galaxy clusters.

Before its untimely demise, Hitomi observed the LMC SNR N132D for only $3.7 \mathrm{ks}$ (Hitomi Collaboration et al 2017), and it readily detected line complexes of $\mathrm{S}$, Ar, and Fe. The Fe emission was highly redshifted at $\sim 800 \mathrm{~km} \mathrm{~s}^{-1}$, but no blueshifted component was detected, suggesting that the Fe-rich ejecta was ejected asymmetrically. In the shorter term (with an anticipated launch date of 2021), the replacement for Hitomi, the X-ray Astronomy Recovery Mission (XARM), offers the chance of $5-7 \mathrm{eV}$ energy resolution over a field-of-view of $\sim 3^{\prime}$.

\footnotetext{
0 See https://heasarc.gsfc.nasa.gov/docs/xarm/
} 
Looking forward to the 2030s, the proposed Lynx X-ray Observatory is intended to have a microcalorimeter with high spatial resolution $\left(1.0^{\prime \prime}\right.$ pixels $)$ over a $5^{\prime}$ field with a few eV energy resolution. These capabilities will enable three-dimensional mapping of the hot metals synthesized in SN explosions, similar to the results presented in Section 3 but at X-ray wavelengths. Additionally, with $\sim 50$ times the effective area of Chandra, Lynx will detect fainter and more distant sources, such as SNRs in Local Group galaxies. In this way Lynx will dramatically increase the sample size of young SNRs with morphological and kinematic measurements. These observations will be crucial to inform SN models and probe SN feedback and enrichment in different Galactic environments.

Acknowledgements LAL gratefully acknowledges that this work was supported through NSF Astronomy \& Astrophysics Grant AST-1517021. The authors thank the organizers of the workshop held in ISSI Bern on 3-7 October 2016 where many helpful discussions took place.

\section{References}

Arzoumanian Z, Chernoff DF, Cordes JM (2002) The Velocity Distribution of Isolated Radio Pulsars. Astrophysical Journal 568:289-301, DOI 10.1086/338805, astro-ph/0106159

Badenes C, Harris J, Zaritsky D, Prieto JL (2009) The Stellar Ancestry of Supernovae in the Magellanic Clouds. I. The Most Recent Supernovae in the Large Magellanic Cloud. Astrophysical Journal 700:727-740, DOI 10.1088/0004-637X/700/1/727, 0902.2787

Badenes C, Maoz D, Draine BT (2010) On the size distribution of supernova remnants in the Magellanic Clouds. Monthly Notices of the Royal Astronomical Society 407:1301-1313, DOI 10.1111/j.1365-2966.2010.17023.x, 1003.3030

Bhalerao J, Park S, Dewey D, Hughes JP, Mori K, Lee JJ (2015) X-Ray Ejecta Kinematics of the Galactic Core-Collapse Supernova Remnant G292.0+1.8. Astrophysical Journal 800:65, DOI 10.1088/0004-637X/800/1/65, 1408.1949

Blondin JM, Lundqvist P, Chevalier RA (1996) Axisymmetric Circumstellar Interaction in Supernovae. Astrophysical Journal 472:257, DOI 10.1086/178060, astro-ph/9601137

Blondin JM, Borkowski KJ, Reynolds SP (2001) Dynamics of Fe Bubbles in Young Supernova Remnants. Astrophysical Journal 557:782-791, DOI 10.1086/321674, astro-ph/0010285

Boggs SE, Harrison FA, Miyasaka H, Grefenstette BW, Zoglauer A, Fryer CL, Reynolds SP, Alexander DM, An H, Barret D, Christensen FE, Craig WW, Forster K, Giommi P, Hailey CJ, Hornstrup A, Kitaguchi T, Koglin JE, Madsen KK, Mao PH, Mori K, Perri M, Pivovaroff MJ, Puccetti S, Rana V, Stern D, Westergaard NJ, Zhang WW (2015) ${ }^{44} \mathrm{Ti}$ gamma-ray emission lines from SN1987A reveal an asymmetric explosion. Science 348:670-671, DOI 10.1126/science.aaa2259

Chakraborti S, Soderberg A, Chomiuk L, Kamble A, Yadav N, Ray A, Hurley K, Margutti R, Milisavljevic D, Bietenholz M, Brunthaler A, Pignata G, Pian E, Mazzali P, Fransson C, Bartel N, Hamuy M, Levesque E, MacFadyen A, Dittmann J, Krauss M, Briggs MS, Connaughton V, Yamaoka K, Takahashi T, Ohno M, Fukazawa Y, Tashiro M, Terada Y, Murakami T, Goldsten J, Barthelmy S, Gehrels N, Cummings J, Krimm H, Palmer D, Golenetskii S, Aptekar R, Frederiks D, Svinkin D, Cline T, Mitrofanov IG, Golovin D, Litvak ML, Sanin AB, Boynton W, Fellows C, Harshman K, Enos H, von Kienlin A, Rau A, Zhang X, Savchenko V (2015) A Missing-link in the Supernova-GRB Connection: The Case of SN 2012ap. Astrophysical Journal 805:187, DOI 10.1088/0004-637X/805/2/187, 1402.6336

Chatterjee S, Vlemmings WHT, Brisken WF, Lazio TJW, Cordes JM, Goss WM, Thorsett SE, Fomalont EB, Lyne AG, Kramer M (2005) Getting Its Kicks: A VLBA Parallax for the Hyperfast Pulsar B1508+55. Astrophysical Journal Letters 630:L61-L64, DOI 10.1086/491701, astro-ph/0509031

Cox DP, Shelton RL, Maciejewski W, Smith RK, Plewa T, Pawl A, Rozyczka M (1999) Modeling W44 as a Supernova Remnant in a Density Gradient with a Partially Formed Dense Shell and Thermal Conduction in the Hot Interior. I. The Analytical Model. Astrophysical Journal 524:179-191, DOI 10.1086/307781 
DeLaney T, Rudnick L, Stage MD, Smith JD, Isensee K, Rho J, Allen GE, Gomez H, Kozasa T, Reach WT, Davis JE, Houck JC (2010) The Three-dimensional Structure of Cassiopeia A. Astrophysical Journal 725:2038-2058, DOI 10.1088/0004-637X/725/2/2038, 1011.3858

Dewey D, Zhekov SA, McCray R, Canizares CR (2008) Chandra HETG Spectra of SN 1987A at 20 Years. Astrophysical Journal Letters 676:L131, DOI 10.1086/587549, 0802.2340

Faucher-Giguère CA, Kaspi VM (2006) Birth and Evolution of Isolated Radio Pulsars. Astrophysical Journal 643:332-355, DOI 10.1086/501516, arXiv:astro-ph/0512585

Fesen RA (2001) An Optical Survey of Outlying Ejecta in Cassiopeia A: Evidence for a Turbulent, Asymmetric Explosion. Astrophysical Journal Supplement Series 133:161-186, DOI $10.1086 / 319181$

Fesen RA, Gunderson KS (1996) Optical Spectroscopy and Imaging of the Northeast Jet in the Cassiopeia A Supernova Remnant. Astrophysical Journal 470:967, DOI 10.1086/177923

Fesen RA, Milisavljevic D (2016) An HST Survey of the Highest-velocity Ejecta in Cassiopeia A. Astrophysical Journal 818:17, DOI 10.3847/0004-637X/818/1/17, 1512.05049

Fesen RA, Hammell MC, Morse J, Chevalier RA, Borkowski KJ, Dopita MA, Gerardy CL, Lawrence SS, Raymond JC, van den Bergh S (2006a) Discovery of Outlying High-Velocity Oxygen-Rich Ejecta in Cassiopeia A. Astrophysical Journal 636:859-872, DOI 10.1086/ 498092, astro-ph/0509067

Fesen RA, Hammell MC, Morse J, Chevalier RA, Borkowski KJ, Dopita MA, Gerardy CL, Lawrence SS, Raymond JC, van den Bergh S (2006b) The Expansion Asymmetry and Age of the Cassiopeia A Supernova Remnant. Astrophysical Journal 645:283-292, DOI 10.1086/504254, astro-ph/0603371

Fesen RA, Zastrow JA, Hammell MC, Shull JM, Silvia DW (2011) Ejecta Knot Flickering, Mass Ablation, and Fragmentation in Cassiopeia A. Astrophysical Journal 736:109, DOI 10.1088/0004-637X/736/2/109, 1105.3970

Filippenko AV (1997) Optical Spectra of Supernovae. Annual Review of Astronomy \& Astrophysics 35:309-355, DOI 10.1146/annurev.astro.35.1.309

Flanagan KA, Canizares CR, Dewey D, Houck JC, Fredericks AC, Schattenburg ML, Markert TH, Davis DS (2004) Chandra High-Resolution X-Ray Spectrum of Supernova Remnant 1E 0102.2-7219. Astrophysical Journal 605:230-246, DOI 10.1086/382145, astro-ph/ 0312509

Fryer CL, Kusenko A (2006) Effects of Neutrino-driven Kicks on the Supernova Explosion Mechanism. Astrophysical Journal Supplement Series 163:335-343, DOI 10.1086/500933, astro-ph/0512033

Gaensler BM (1998) The Nature of Bilateral Supernova Remnants. Astrophysical Journal 493:781-792, DOI 10.1086/305146, astro-ph/9707149

Gaensler BM, Slane PO (2006) The Evolution and Structure of Pulsar Wind Nebulae. Annual Review of Astronomy \& Astrophysics 44:17-47, DOI 10.1146/annurev.astro.44.051905. 092528, astro-ph/0601081

Garofali K, Williams BF, Plucinsky PP, Gaetz TJ, Wold B, Haberl F, Long KS, Blair WP, Pannuti TG, Winkler PF, Gross J (2017) Supernova remnants in M33: X-ray properties as observed by XMM-Newton. Monthly Notices of the Royal Astronomical Society 472:308333, DOI 10.1093/mnras/stx1905, 1708.01239

González-Casanova DF, De Colle F, Ramirez-Ruiz E, Lopez LA (2014) The Morphology and Dynamics of Jet-driven Supernova Remnants: The Case of W49B. Astrophysical Journal Letters 781:L26, DOI 10.1088/2041-8205/781/2/L26, 1309.7049

Green DA (2014) A catalogue of 294 Galactic supernova remnants. Bulletin of the Astronomical Society of India 42:47-58, 1409.0637

Grefenstette BW, Harrison FA, Boggs SE, Reynolds SP, Fryer CL, Madsen KK, Wik DR, Zoglauer A, Ellinger CI, Alexander DM, An H, Barret D, Christensen FE, Craig WW, Forster K, Giommi P, Hailey CJ, Hornstrup A, Kaspi VM, Kitaguchi T, Koglin JE, Mao PH, Miyasaka H, Mori K, Perri M, Pivovaroff MJ, Puccetti S, Rana V, Stern D, Westergaard NJ, Zhang WW (2014) Asymmetries in core-collapse supernovae from maps of radioactive ${ }^{44} \mathrm{Ti}$ in CassiopeiaA. Nature 506:339-342, DOI 10.1038/nature12997, 1403.4978

Grefenstette BW, Fryer CL, Harrison FA, Boggs SE, DeLaney T, Laming JM, Reynolds SP, Alexander DM, Barret D, Christensen FE, Craig WW, Forster K, Giommi P, Hailey CJ, Hornstrup A, Kitaguchi T, Koglin JE, Lopez L, Mao PH, Madsen KK, Miyasaka H, Mori K, Perri M, Pivovaroff MJ, Puccetti S, Rana V, Stern D, Westergaard NJ, Wik DR, Zhang WW, Zoglauer A (2017) The Distribution of Radioactive ${ }^{44} \mathrm{Ti}$ in Cassiopeia A. Astrophysical Journal 834:19, DOI 10.3847/1538-4357/834/1/19, 1612.02774 
Hammer NJ, Janka HT, Müller E (2010) Three-dimensional Simulations of Mixing Instabilities in Supernova Explosions. Astrophysical Journal 714:1371-1385, DOI 10.1088/0004-637X/ $714 / 2 / 1371,0908.3474$

Harrison FA, Craig WW, Christensen FE, Hailey CJ, Zhang WW, Boggs SE, Stern D, Cook WR, Forster K, Giommi P, Grefenstette BW, Kim Y, Kitaguchi T, Koglin JE, Madsen KK, Mao PH, Miyasaka H, Mori K, Perri M, Pivovaroff MJ, Puccetti S, Rana VR, Westergaard NJ, Willis J, Zoglauer A, An H, Bachetti M, Barrière NM, Bellm EC, Bhalerao V, Brejnholt NF, Fuerst F, Liebe CC, Markwardt CB, Nynka M, Vogel JK, Walton DJ, Wik DR, Alexander DM, Cominsky LR, Hornschemeier AE, Hornstrup A, Kaspi VM, Madejski GM, Matt G, Molendi S, Smith DM, Tomsick JA, Ajello M, Ballantyne DR, Baloković M, Barret D, Bauer FE, Blandford RD, Brandt WN, Brenneman LW, Chiang J, Chakrabarty D, Chenevez J, Comastri A, Dufour F, Elvis M, Fabian AC, Farrah D, Fryer CL, Gotthelf EV, Grindlay JE, Helfand DJ, Krivonos R, Meier DL, Miller JM, Natalucci L, Ogle P, Ofek EO, Ptak A, Reynolds SP, Rigby JR, Tagliaferri G, Thorsett SE, Treister E, Urry CM (2013) The Nuclear Spectroscopic Telescope Array (NuSTAR) High-energy X-Ray Mission. Astrophysical Journal 770:103, DOI 10.1088/0004-637X/770/2/103, 1301.7307

Hitomi Collaboration, Aharonian F, Akamatsu H, Akimoto F, Allen SW, Angelini L, Audard M, Awaki H, Axelsson M, Bamba A, Bautz MW, Blandford R, Brenneman LW, Brown GV, Bulbul E, Cackett EM, Chernyakova M, Chiao MP, Coppi PS, Costantini E, de Plaa J, de Vries CP, den Herder JW, Done C, Dotani T, Ebisawa K, Eckart ME, Enoto T, Ezoe Y, Fabian AC, Ferrigno C, Foster AR, Fujimoto R, Fukazawa Y, Furuzawa A, Galeazzi M, Gallo LC, Gandhi P, Giustini M, Goldwurm A, Gu L, Guainazzi M, Haba Y, Hagino K, Hamaguchi K, Harrus IM, Hatsukade I, Hayashi K, Hayashi T, Hayashida K, Hiraga JS, Hornschemeier A, Hoshino A, Hughes JP, Ichinohe Y, Iizuka R, Inoue H, Inoue Y, Ishida M, Ishikawa K, Ishisaki Y, Iwai M, Kaastra J, Kallman T, Kamae T, Kataoka J, Katsuda S, Kawai N, Kelley RL, Kilbourne CA, Kitaguchi T, Kitamoto S, Kitayama T, Kohmura T, Kokubun M, Koyama K, Koyama S, Kretschmar P, Krimm HA, Kubota A, Kunieda H, Laurent P, Lee SH, Leutenegger MA, Limousin O, Loewenstein M, Long KS, Lumb D, Madejski G, Maeda Y, Maier D, Makishima K, Markevitch M, Matsumoto H, Matsushita K, McCammon D, McNamara BR, Mehdipour M, Miller ED, Miller JM, Mineshige S, Mitsuda K, Mitsuishi I, Miyazawa T, Mizuno T, Mori H, Mori K, Mukai K, Murakami H, Mushotzky RF, Nakagawa T, Nakajima H, Nakamori T, Nakashima S, Nakazawa K, Nobukawa KK, Nobukawa M, Noda H, Odaka H, Ohashi T, Ohno M, Okajima T, Ota N, Ozaki M, Paerels F, Paltani S, Petre R, Pinto C, Porter FS, Pottschmidt K, Reynolds CS, Safi-Harb S, Saito S, Sakai K, Sasaki T, Sato G, Sato K, Sato R, Sato T, Sawada M, Schartel N, Serlemtsos PJ, Seta H, Shidatsu M, Simionescu A, Smith RK, Soong Y, Stawarz E, Sugawara Y, Sugita S, Szymkowiak A, Tajima H, Takahashi H, Takahashi T, Takeda S, Takei Y, Tamagawa T, Tamura T, Tanaka T, Tanaka Y, Tanaka YT, Tashiro MS, Tawara Y, Terada Y, Terashima Y, Tombesi F, Tomida H, Tsuboi Y, Tsujimoto M, Tsunemi H, Tsuru TG, Uchida H, Uchiyama H, Uchiyama Y, Ueda S, Ueda Y, Uno S, Urry CM, Ursino E, Watanabe S, Werner N, Wilkins DR, Williams BJ, Yamada S, Yamaguchi H, Yamaoka K, Yamasaki NY, Yamauchi M, Yamauchi S, Yaqoob T, Yatsu Y, Yonetoku D, Zhuravleva I, Zoghbi A (2017) Hitomi Observations of the LMC SNR N132D: Highly Redshifted X-ray Emission from Iron Ejecta. ArXiv e-prints 1712.02365

Hobbs G, Lorimer DR, Lyne AG, Kramer M (2005) A statistical study of 233 pulsar proper motions. Monthly Notices of the Royal Astronomical Society 360:974-992, DOI 10.1111/ j.1365-2966.2005.09087.x, astro-ph/0504584

Holland-Ashford T, Lopez LA, Auchettl K, Temim T, Ramirez-Ruiz E (2017) Comparing Neutron Star Kicks to Supernova Remnant Asymmetries. Astrophysical Journal 844:84, DOI 10.3847/1538-4357/aa7a5c, 1705.08454

Holoien TWS, Stanek KZ, Kochanek CS, Shappee BJ, Prieto JL, Brimacombe J, Bersier D, Bishop DW, Dong S, Brown JS, Danilet AB, Simonian GV, Basu U, Beacom JF, Falco E, Pojmanski G, Skowron DM, Woźniak PR, Ávila CG, Conseil E, Contreras C, Cruz I, Fernández JM, Koff RA, Guo Z, Herczeg GJ, Hissong J, Hsiao EY, Jose J, Kiyota S, Long F, Monard LAG, Nicholls B, Nicolas J, Wiethoff WS (2017) The ASAS-SN bright supernova catalogue - I. 2013-2014. Monthly Notices of the Royal Astronomical Society 464:2672-2686, DOI 10.1093/mnras/stw2273, 1604.00396

Hughes JP, Rakowski CE, Burrows DN, Slane PO (2000) Nucleosynthesis and Mixing in Cassiopeia A. Astrophysical Journal Letters 528:L109-L113, DOI 10.1086/312438, astro-ph/9910474 
Hwang U, Hughes JP, Petre R (1998) The X-Ray Iron Emission from Tycho's Supernova Remnant. Astrophysical Journal 497:833, DOI 10.1086/305501, arXiv:astro-ph/9712241

Hwang U, Holt SS, Petre R (2000) Mapping the X-Ray-emitting Ejecta in Cassiopeia A with Chandra. Astrophysical Journal Letters 537:L119-L122, DOI 10.1086/312776, astro-ph/ 0005560

Isensee K, Rudnick L, DeLaney T, Smith JD, Rho J, Reach WT, Kozasa T, Gomez H (2010) The Three-dimensional Structure of Interior Ejecta in Cassiopeia A at High Spectral Resolution. Astrophysical Journal 725:2059-2070, DOI 10.1088/0004-637X/725/2/2059, 1006.0918

Itoh H, Masai K (1989) The effect of a circumstellar medium on the X-ray emission of young remnants of Type II supernovae. Monthly Notices of the Royal Astronomical Society 236:885-899, DOI 10.1093/mnras/236.4.885

Janka HT (2017) Neutron Star Kicks by the Gravitational Tug-boat Mechanism in Asymmetric Supernova Explosions: Progenitor and Explosion Dependence. Astrophysical Journal 837:84, DOI 10.3847/1538-4357/aa618e, 1611.07562

Janka HT, Melson T, Summa A (2016) Physics of Core-Collapse Supernovae in Three Dimensions: A Sneak Preview. Annual Review of Nuclear and Particle Science 66:341-375, DOI 10.1146/annurev-nucl-102115-044747, 1602.05576

Jeltema TE, Canizares CR, Bautz MW, Buote DA (2005) The Evolution of Structure in X-Ray Clusters of Galaxies. Astrophysical Journal 624:606-629, DOI 10.1086/428940, astro-ph/ 0501360

Jennings ZG, Williams BF, Murphy JW, Dalcanton JJ, Gilbert KM, Dolphin AE, Fouesneau M, Weisz DR (2012) Supernova Remnant Progenitor Masses in M31. Astrophysical Journal 761:26, DOI 10.1088/0004-637X/761/1/26, 1210.6353

Kaplan DL, Chatterjee S, Gaensler BM, Anderson J (2008) A Precise Proper Motion for the Crab Pulsar, and the Difficulty of Testing Spin-Kick Alignment for Young Neutron Stars. Astrophysical Journal 677:1201-1215, DOI 10.1086/529026, 0801.1142

Kaspi VM (1998) Radio pulsar/supernova remnant associations. Advances in Space Research 21:167-176, DOI 10.1016/S0273-1177(97)00801-6

Katsuda S, Morii M, Janka HT, Wongwathanarat A, Nakamura K, Kotake K, Mori K, Müller E, Takiwaki T, Tanaka M, Tominaga N, Tsunemi H (2017) Intermediate-Mass-Elements in Young Supernova Remnants Reveal Neutron Star Kicks by Asymmetric Explosions. ArXiv e-prints 1710.10372

Kawasaki M, Ozaki M, Nagase F, Inoue H, Petre R (2005) Ionization States and Plasma Structures of Mixed-Morphology Supernova Remnants Observed with ASCA. Astrophysical Journal 631:935-946, DOI 10.1086/432591, astro-ph/0507348

Kawasaki MT, Ozaki M, Nagase F, Masai K, Ishida M, Petre R (2002) ASCA Observations of the Supernova Remnant IC 443: Thermal Structure and Detection of Overionized Plasma. Astrophysical Journal 572:897-905, DOI 10.1086/340383, astro-ph/0202484

Krause O, Birkmann SM, Usuda T, Hattori T, Goto M, Rieke GH, Misselt KA (2008) The Cassiopeia A Supernova Was of Type IIb. Science 320:1195, DOI 10.1126/science.1155788, 0805.4557

Lacey CK, Lazio TJW, Kassim NE, Duric N, Briggs DS, Dyer KK (2001) Spatially Resolved Thermal Continuum Absorption against Supernova Remnant W49B. Astrophysical Journal 559:954-962, DOI 10.1086/322372, astro-ph/0105451

Lai D (2001) Neutron Star Kicks and Asymmetric Supernovae. In: Blaschke D, Glendenning NK, Sedrakian A (eds) Physics of Neutron Star Interiors, Lecture Notes in Physics, Berlin Springer Verlag, vol 578, p 424, astro-ph/0012049

Law NM, Kulkarni SR, Dekany RG, Ofek EO, Quimby RM, Nugent PE, Surace J, Grillmair CC, Bloom JS, Kasliwal MM, Bildsten L, Brown T, Cenko SB, Ciardi D, Croner E, Djorgovski SG, van Eyken J, Filippenko AV, Fox DB, Gal-Yam A, Hale D, Hamam N, Helou G, Henning J, Howell DA, Jacobsen J, Laher R, Mattingly S, McKenna D, Pickles A, Poznanski D, Rahmer G, Rau A, Rosing W, Shara M, Smith R, Starr D, Sullivan M, Velur V, Walters R, Zolkower J (2009) The Palomar Transient Factory: System Overview, Performance, and First Results. Publications of the Astronomical Society of the Pacific 121:1395, DOI 10.1086/648598, 0906.5350

Lawrence SS, MacAlpine GM, Uomoto A, Woodgate BE, Brown LW, Oliversen RJ, Lowenthal JD, Liu C (1995) Three-Dimensional Fabry-Perot Imaging Spectroscopy of the Crab Nebula, Cassiopeia A, and Nova GK Persei. Astronomical Journal 109:2635, DOI 10.1086/117477 
Lazendic JS, Dewey D, Schulz NS, Canizares CR (2006) The Kinematic and Plasma Properties of X-Ray Knots in Cassiopeia A from the Chandra HETGS. Astrophysical Journal 651:250267, DOI 10.1086/507481, astro-ph/0605078

Leaman J, Li W, Chornock R, Filippenko AV (2011) Nearby supernova rates from the Lick Observatory Supernova Search - I. The methods and data base. Monthly Notices of the Royal Astronomical Society 412:1419-1440, DOI 10.1111/j.1365-2966.2011.18158.x, 1006. 4611

Lentz EJ, Bruenn SW, Hix WR, Mezzacappa A, Messer OEB, Endeve E, Blondin JM, Harris JA, Marronetti P, Yakunin KN (2015) Three-dimensional Core-collapse Supernova Simulated Using a $15 \mathrm{M}$ ? Progenitor. Astrophysical Journal Letters 807:L31, DOI 10.1088/2041-8205/807/2/L31, 1505.05110

Leonard DC, Filippenko AV, Ganeshalingam M, Serduke FJD, Li W, Swift BJ, Gal-Yam A, Foley RJ, Fox DB, Park S, Hoffman JL, Wong DS (2006) A non-spherical core in the explosion of supernova SN 2004dj. Nature 440:505-507, DOI 10.1038/nature04558, arXiv:astro-ph/0603297

Lopez LA (2014) What Shapes Supernova Remnants? In: Ray A, McCray RA (eds) Supernova Environmental Impacts, IAU Symposium, vol 296, pp 239-244, DOI 10.1017/ S1743921313009538, 1306.2638

Lopez LA, Ramirez-Ruiz E, Badenes C, Huppenkothen D, Jeltema TE, Pooley DA (2009a) Typing Supernova Remnants Using X-Ray Line Emission Morphologies. Astrophysical Journal Letters 706:L106-L109, DOI 10.1088/0004-637X/706/1/L106, 0910.3208

Lopez LA, Ramirez-Ruiz E, Pooley DA, Jeltema TE (2009b) Tools for Dissecting Supernova Remnants Observed with Chandra: Methods and Application to the Galactic Remnant W49B. Astrophysical Journal 691:875-893, DOI 10.1088/0004-637X/691/1/875, 0810.0009

Lopez LA, Ramirez-Ruiz E, Huppenkothen D, Badenes C, Pooley DA (2011) Using the X-ray Morphology of Young Supernova Remnants to Constrain Explosion Type, Ejecta Distribution, and Chemical Mixing. Astrophysical Journal 732:114, DOI 10.1088/0004-637X/ $732 / 2 / 114,1011.0731$

Lopez LA, Pearson S, Ramirez-Ruiz E, Castro D, Yamaguchi H, Slane PO, Smith RK (2013a) Unraveling the Origin of Overionized Plasma in the Galactic Supernova Remnant W49B. Astrophysical Journal 777:145, DOI 10.1088/0004-637X/777/2/145, 1309.1464

Lopez LA, Ramirez-Ruiz E, Castro D, Pearson S (2013b) The Galactic Supernova Remnant W49B Likely Originates from a Jet-driven, Core-collapse Explosion. Astrophysical Journal 764:50, DOI 10.1088/0004-637X/764/1/50, 1301.0618

Lopez LA, Castro D, Slane PO, Ramirez-Ruiz E, Badenes C (2014) Identification of a Jetdriven Supernova Remnant in the Small Magellanic Cloud: Possible Evidence for the Enhancement of Bipolar Explosions at Low Metallicity. Astrophysical Journal 788:5, DOI 10.1088/0004-637X/788/1/5, 1310.4498

Lyne AG, Lorimer DR (1994) High birth velocities of radio pulsars. Nature 369:127-129, DOI $10.1038 / 369127 \mathrm{a} 0$

Maeda K, Nomoto K (2003) Bipolar Supernova Explosions: Nucleosynthesis and Implications for Abundances in Extremely Metal-Poor Stars. Astrophysical Journal 598:1163-1200, DOI 10.1086/378948, arXiv:astro-ph/0304172

Maggi P, Haberl F, Kavanagh PJ, Sasaki M, Bozzetto LM, Filipović MD, Vasilopoulos G, Pietsch W, Points SD, Chu YH, Dickel J, Ehle M, Williams R, Greiner J (2016) The population of X-ray supernova remnants in the Large Magellanic Cloud. Astronomy \& Astrophysics 585:A162, DOI 10.1051/0004-6361/201526932, 1509.09223

Mathewson DS, Ford VL, Dopita MA, Tuohy IR, Long KS, Helfand DJ (1983) Supernova remnants in the Magellanic Clouds. Astrophysical Journal Supplement Series 51:345-355, DOI 10.1086/190854

Mazzali PA, Kawabata KS, Maeda K, Foley RJ, Nomoto K, Deng J, Suzuki T, Iye M, Kashikawa N, Ohyama Y, Filippenko AV, Qiu Y, Wei J (2007) The Aspherical Properties of the Energetic Type Ic SN 2002ap as Inferred from Its Nebular Spectra. Astrophysical Journal 670:592-599, DOI 10.1086/521873, 0708.0966

McCray R, Fransson C (2016) The Remnant of Supernova 1987A. Annual Review of Astronomy \& Astrophysics 54:19-52, DOI 10.1146/annurev-astro-082615-105405

Milisavljevic D, Fesen RA (2013) A Detailed Kinematic Map of Cassiopeia A's Optical Main Shell and Outer High-velocity Ejecta. Astrophysical Journal 772:134, DOI 10.1088/0004-637X/772/2/134, 1306.2310 
Milisavljevic D, Fesen RA (2015) The bubble-like interior of the core-collapse supernova remnant Cassiopeia A. Science 347:526-530, DOI 10.1126/science.1261949, 1501.07283

Milisavljevic D, Fesen RA, Chevalier RA, Kirshner RP, Challis P, Turatto M (2012) Latetime Optical Emission from Core-collapse Supernovae. Astrophysical Journal 751:25, DOI 10.1088/0004-637X/751/1/25, 1203.0006

Minkowski R (1941) Spectra of Supernovae. Publications of the Astronomical Society of the Pacific 53:224-+, DOI 10.1086/125315

Modjaz M, Kewley L, Kirshner RP, Stanek KZ, Challis P, Garnavich PM, Greene JE, Kelly PL, Prieto JL (2008) Measured Metallicities at the Sites of Nearby Broad-Lined Type Ic Supernovae and Implications for the Supernovae Gamma-Ray Burst Connection. Astronomical Journal 135:1136-1150, DOI 10.1088/0004-6256/135/4/1136, astro-ph/0701246

Moriya TJ (2012) Progenitors of Recombining Supernova Remnants. Astrophysical Journal Letters 750:L13, DOI 10.1088/2041-8205/750/1/L13, 1203.5799

Müller B, Melson T, Heger A, Janka HT (2017) Supernova simulations from a 3D progenitor model - Impact of perturbations and evolution of explosion properties. Monthly Notices of the Royal Astronomical Society 472:491-513, DOI 10.1093/mnras/stx1962, 1705.00620

Nugent RL (1998) New Measurements of the Expansion of the Crab Nebula. Publications of the Astronomical Society of the Pacific 110:831-836, DOI 10.1086/316199

Orlando S, Bocchino F, Reale F, Peres G, Petruk O (2007) On the origin of asymmetries in bilateral supernova remnants. Astronomy \& Astrophysics 470:927-939, DOI 10.1051/ 0004-6361:20066045, 0704.0890

Ozawa M, Koyama K, Yamaguchi H, Masai K, Tamagawa T (2009) Suzaku Discovery of the Strong Radiative Recombination Continuum of Iron from the Supernova Remnant W49B. Astrophysical Journal Letters 706:L71-L75, DOI 10.1088/0004-637X/706/1/L71, 0910.3302

Patnaude DJ, Fesen RA (2014) A Comparison of X-Ray and Optical Emission in Cassiopeia A. Astrophysical Journal 789:138, DOI 10.1088/0004-637X/789/2/138, 1403.5812

Pavlov GG, Zavlin VE, Aschenbach B, Trümper J, Sanwal D (2000) The Compact Central Object in Cassiopeia A: A Neutron Star with Hot Polar Caps or a Black Hole? Astrophysical Journal Letters 531:L53-L56, DOI 10.1086/312521, astro-ph/9912024

Peters CL, Lopez LA, Ramirez-Ruiz E, Stassun KG, Figueroa-Feliciano E (2013) Constraining Explosion Type of Young Supernova Remnants Using $24 \mu \mathrm{m}$ Emission Morphology. Astrophysical Journal Letters 771:L38, DOI 10.1088/2041-8205/771/2/L38, 1306.3503

Reed JE, Hester JJ, Fabian AC, Winkler PF (1995) The Three-dimensional Structure of the Cassiopeia A Supernova Remnant. I. The Spherical Shell. Astrophysical Journal 440:706, DOI 10.1086/175308

Rest A, Suntzeff NB, Olsen K, Prieto JL, Smith RC, Welch DL, Becker A, Bergmann M, Clocchiatti A, Cook K, Garg A, Huber M, Miknaitis G, Minniti D, Nikolaev S, Stubbs C (2005) Light echoes from ancient supernovae in the Large Magellanic Cloud. Nature 438:1132-1134, DOI 10.1038/nature04365, astro-ph/0510738

Rest A, Matheson T, Blondin S, Bergmann M, Welch DL, Suntzeff NB, Smith RC, Olsen K, Prieto JL, Garg A, Challis P, Stubbs C, Hicken M, Modjaz M, Wood-Vasey WM, Zenteno A, Damke G, Newman A, Huber M, Cook KH, Nikolaev S, Becker AC, Miceli A, Covarrubias R, Morelli L, Pignata G, Clocchiatti A, Minniti D, Foley RJ (2008) Spectral Identification of an Ancient Supernova Using Light Echoes in the Large Magellanic Cloud. Astrophysical Journal 680:1137-1148, DOI 10.1086/587158, 0801.4762

Rest A, Foley RJ, Sinnott B, Welch DL, Badenes C, Filippenko AV, Bergmann M, Bhatti WA, Blondin S, Challis P, Damke G, Finley H, Huber ME, Kasen D, Kirshner RP, Matheson T, Mazzali P, Minniti D, Nakajima R, Narayan G, Olsen K, Sauer D, Smith RC, Suntzeff NB (2011) Direct Confirmation of the Asymmetry of the Cas A Supernova with Light Echoes. Astrophysical Journal 732:3, DOI 10.1088/0004-637X/732/1/3, 1003.5660

Rho J, Petre R (1998) Mixed-Morphology Supernova Remnants. Astrophysical Journal Letters 503:L167-L170, DOI 10.1086/311538

Roberts LF, Ott CD, Haas R, O'Connor EP, Diener P, Schnetter E (2016) General-Relativistic Three-Dimensional Multi-group Neutrino Radiation-Hydrodynamics Simulations of CoreCollapse Supernovae. Astrophysical Journal 831:98, DOI 10.3847/0004-637X/831/1/98, 1604.07848

Sasaki M, Pietsch W, Haberl F, Hatzidimitriou D, Stiele H, Williams B, Kong A, Kolb U (2012) Supernova remnants and candidates detected in the XMM-Newton M 31 large survey. Astronomy \& Astrophysics 544:A144, DOI 10.1051/0004-6361/201219025, 1206.4789 
Scheck L, Kifonidis K, Janka HT, Müller E (2006) Multidimensional supernova simulations with approximative neutrino transport. I. Neutron star kicks and the anisotropy of neutrino-driven explosions in two spatial dimensions. Astronomy \& Astrophysics 457:963986, DOI 10.1051/0004-6361:20064855, astro-ph/0601302

Seward FD (1990) Einstein Observations of Galactic supernova remnants. Astrophysical Journal Supplement Series 73:781-819, DOI 10.1086/191489

Seward FD, Tucker WH, Fesen RA (2006) Faint X-Ray Structure in the Crab Pulsar Wind Nebula. Astrophysical Journal 652:1277-1287, DOI 10.1086/508532, astro-ph/0608485

Shelton RL, Cox DP, Maciejewski W, Smith RK, Plewa T, Pawl A, Rozyczka M (1999) Modeling W44 as a Supernova Remnant in a Density Gradient with a Partially Formed Dense Shell and Thermal Conduction in the Hot Interior. II. The Hydrodynamic Models. Astrophysical Journal 524:192-212, DOI 10.1086/307799, astro-ph/9806090

Shimizu T, Masai K, Koyama K (2012) Evolution of Supernova Remnants Expanding out of the Dense Circumstellar Matter into the Rarefied Interstellar Medium. Publications of the Astronomical Society of Japan 64:24, DOI 10.1093/pasj/64.2.24, 1110.4948

Soderberg AM, Chakraborti S, Pignata G, Chevalier RA, Chandra P, Ray A, Wieringa MH, Copete A, Chaplin V, Connaughton V, Barthelmy SD, Bietenholz MF, Chugai N, Stritzinger MD, Hamuy M, Fransson C, Fox O, Levesque EM, Grindlay JE, Challis P, Foley RJ, Kirshner RP, Milne PA, Torres MAP (2010) A relativistic type Ibc supernova without a detected $\gamma$-ray burst. Nature 463:513-515, DOI 10.1038/nature08714, 0908.2817

Temim T, Slane P, Castro D, Plucinsky PP, Gelfand J, Dickel JR (2013) High-energy Emission from the Composite Supernova Remnant MSH 15-56. Astrophysical Journal 768:61, DOI 10.1088/0004-637X/768/1/61, 1303.2425

Thorstensen JR, Fesen RA, van den Bergh S (2001) The Expansion Center and Dynamical Age of the Galactic Supernova Remnant Cassiopeia A. Astronomical Journal 122:297-307, DOI 10.1086/321138, astro-ph/0104188

Uchida H, Koyama K, Yamaguchi H, Sawada M, Ohnishi T, Tsuru TG, Tanaka T, Yoshiike S, Fukui Y (2012) Recombining Plasma and Hard X-Ray Filament in the Mixed-Morphology Supernova Remnant W 44. Publications of the Astronomical Society of Japan 64:141, DOI 10.1093/pasj/64.6.141, 1208.0113

Ugliano M, Janka HT, Marek A, Arcones A (2012) Progenitor-explosion Connection and Remnant Birth Masses for Neutrino-driven Supernovae of Iron-core Progenitors. Astrophysical Journal 757:69, DOI 10.1088/0004-637X/757/1/69, 1205.3657

Utrobin VP, Wongwathanarat A, Janka HT, Müller E (2017) Light-curve Analysis of Ordinary Type IIP Supernovae Based on Neutrino-driven Explosion Simulations in Three Dimensions. Astrophysical Journal 846:37, DOI 10.3847/1538-4357/aa8594, 1704.03800

Vink J (2012) Supernova remnants: the X-ray perspective. The Astronomy \& Astrophysics Review 20:49, DOI 10.1007/s00159-011-0049-1, 1112.0576

Wang CY, Chevalier RA (2001) Instabilities and Clumping in Type IA Supernova Remnants. Astrophysical Journal 549:1119-1134, DOI 10.1086/319439, astro-ph/0005105

Wang L, Wheeler JC (2008) Spectropolarimetry of Supernovae. Annual Review of Astronomy \& Astrophysics 46:433-474, DOI 10.1146/annurev.astro.46.060407.145139, 0811.1054

Wardle M, Yusef-Zadeh F (2002) Supernova Remnant OH Masers: Signposts of Cosmic Collision. Science 296:2350-2354, DOI 10.1126/science.1068168

West JL, Safi-Harb S, Jaffe T, Kothes R, Landecker TL, Foster T (2016) The connection between supernova remnants and the Galactic magnetic field: A global radio study of the axisymmetric sample. Astronomy \& Astrophysics 587:A148, DOI 10.1051/0004-6361/ 201527001, 1510.08536

West JL, Safi-Harb S, Ferrand G (2017) The connection between supernova remnants and the Galactic magnetic field: An analysis of quasi-parallel and quasi-perpendicular cosmic-ray acceleration for the axisymmetric sample. Astronomy \& Astrophysics 597:A121, DOI 10.1051/0004-6361/201628079, 1609.02887

Williams RM, Chu YH, Dickel JR, Petre R, Smith RC, Tavarez M (1999) Supernova Remnants in the Magellanic Clouds. III. An X-Ray Atlas of Large Magellanic Cloud Supernova Remnants. Astrophysical Journal Supplement Series 123:467-485, DOI 10.1086/313246, astro-ph/9905073

Willingale R, Bleeker JAM, van der Heyden KJ, Kaastra JS, Vink J (2002) X-ray spectral imaging and Doppler mapping of Cassiopeia A. Astronomy \& Astrophysics 381:1039-1048, DOI 10.1051/0004-6361:20011614, astro-ph/0107270

Winkler PF, Petre R (2007) Direct Measurement of Neutron Star Recoil in the Oxygen-rich 
Supernova Remnant Puppis A. Astrophysical Journal 670:635-642, DOI 10.1086/522101, astro-ph/0608205

Winkler PF, Williams BJ, Reynolds SP, Petre R, Long KS, Katsuda S, Hwang U (2014) A High-resolution X-Ray and Optical Study of SN 1006: Asymmetric Expansion and Smallscale Structure in a Type Ia Supernova Remnant. Astrophysical Journal 781:65, DOI 10.1088/0004-637X/781/2/65, 1312.3367

Wongwathanarat A, Janka HT, Müller E (2013a) Three-dimensional neutrino-driven supernovae: Neutron star kicks, spins, and asymmetric ejection of nucleosynthesis products. Astronomy \& Astrophysics 552:A126, DOI 10.1051/0004-6361/201220636, 1210.8148

Wongwathanarat A, Janka HT, Müller E (2013b) Three-dimensional neutrino-driven supernovae: Neutron star kicks, spins, and asymmetric ejection of nucleosynthesis products. Astronomy \& Astrophysics 552:A126, DOI 10.1051/0004-6361/201220636, 1210.8148

Wongwathanarat A, Janka HT, Müller E, Pllumbi E, Wanajo S (2017) Production and Distribution of ${ }^{44} \mathrm{Ti}$ and ${ }^{56} \mathrm{Ni}$ in a Three-dimensional Supernova Model Resembling Cassiopeia A. Astrophysical Journal 842:13, DOI 10.3847/1538-4357/aa72de, 1610.05643

Woosley SE, Heger A, Weaver TA (2002) The evolution and explosion of massive stars. Reviews of Modern Physics 74:1015-1071, DOI 10.1103/RevModPhys.74.1015

Yamaguchi H, Ozawa M, Koyama K, Masai K, Hiraga JS, Ozaki M, Yonetoku D (2009) Discovery of Strong Radiative Recombination Continua from the Supernova Remnant IC 443 with Suzaku. Astrophysical Journal Letters 705:L6-L9, DOI 10.1088/0004-637X/705/ 1/L6, 0909.3848

Yamaguchi H, Badenes C, Petre R, Nakano T, Castro D, Enoto T, Hiraga JS, Hughes JP, Maeda Y, Nobukawa M, Safi-Harb S, Slane PO, Smith RK, Uchida H (2014) Discriminating the Progenitor Type of Supernova Remnants with Iron K-shell Emission. Astrophysical Journal Letters 785:L27, DOI 10.1088/2041-8205/785/2/L27, 1403.5154

Young PA, Fryer CL, Hungerford A, Arnett D, Rockefeller G, Timmes FX, Voit B, Meakin C, Eriksen KA (2006) Constraints on the Progenitor of Cassiopeia A. Astrophysical Journal 640:891-900, DOI 10.1086/500108, astro-ph/0511806 